\title{
Dark solitons in external potentials
}

\author{
Dmitry E. Pelinovsky ${ }^{\dagger}$ and Panayotis G. Kevrekidis ${ }^{\dagger \dagger}$ \\ $\dagger$ Department of Mathematics, McMaster University, Hamilton, Ontario, Canada, L8S 4K1 \\ $\dagger \dagger$ Department of Mathematics and Statistics, University of Massachusetts, Amherst, MA 01003
}

August 11, 2018

\begin{abstract}
We consider the persistence and stability of dark solitons in the Gross-Pitaevskii (GP) equation with a small decaying potential. We show that families of black solitons with zero speed originate from extremal points of an appropriately defined effective potential and persist for sufficiently small strength of the potential. We prove that families at the maximum points are generally unstable with exactly one real positive eigenvalue, while families at the minimum points are generally unstable with exactly two complex-conjugated eigenvalues with positive real part. This mechanism of destabilization of the black soliton is confirmed in numerical approximations of eigenvalues of the linearized GP equation and full numerical simulations of the nonlinear GP equation with cubic nonlinearity. We illustrate the monotonic instability associated with the real eigenvalues and the oscillatory instability associated with the complex eigenvalues and compare the numerical results of evolution of a dark soliton with the predictions of Newton's particle law for its position.
\end{abstract}

\section{Introduction}

Dark solitons are solutions of nonlinear PDEs in the space of one dimension with non-zero boundary conditions and non-zero phase shift. They are represented by a family of traveling wave solutions extending from the limit of zero speed (so-called black solitons) to the limit of sound speed (so-called grey solitons). From a physical point of view, dark solitons are waves in defocusing nonlinear systems which move along the modulationally stable continuous-wave background.

The original interest in studies of dark solitons emerged, roughly, two decades ago in the context of nonlinear optics, where dark solitons provide modulations of the light intensity of an optical beam traveling in a planar waveguide [22. The main model for dark solitons in nonlinear optics is the generalized nonlinear Schrödinger (NLS) equation

$$
i u_{t}=-\frac{1}{2} u_{x x}+f\left(|u|^{2}\right) u
$$

where $u(x, t): \mathbb{R} \times \mathbb{R}_{+} \mapsto \mathbb{C}$ and $f(q): \mathbb{R}_{+} \rightarrow \mathbb{R}$. We assume that $f(q)$ is a smooth monotonically increasing function on $q \in \mathcal{I} \subset \mathbb{R}_{+}$. Particular examples of $f(q)$ include the cubic NLS with $f(q)=q$, the cubic-quintic NLS with $f(q)=\alpha q+q^{2}, \alpha \in \mathbb{R}$ and the saturable NLS with $f=-1 /(1+\beta q)$, $\beta \in \mathbb{R}_{+}$. 
Among others, several analytical results were important in the development of dark solitons in recent years: perturbation theory based on renormalized power [24] and momentum [23], orbital stability of dark solitons [1, 2, completeness of eigenfunctions in the cubic NLS [8, 15, inverse scattering for the vector cubic NLS equation [41, construction of the Evans function for dark solitons in the perturbed cubic NLS [19, asymptotic analysis of the radiation and dynamics of dark solitons [26, 27, 35, and spectral analysis of transverse instabilities of one-dimensional dark solitons [25, 36].

Subsequently, rigorous analysis of the existence and stability of dark solitons was developed in the last decade based on the earlier physical literature. In particular, Zhidkov [46] proved local existence of the Cauchy problem and stability of kink solutions, de Bouard [4] proved spectral and nonlinear instability of stationary bubbles (black solitons with zero phase shift), Lin 28 proved the criterion for orbital stability and instability of dark solitons (for non-zero velocities), Maris 29] studied bifurcation of dark solitons (for non-zero velocities) in the presence of delta-function potential and its generalizations, and Di Menza and Gallo 31] proved recently the stability criterion for kinks (black solitons with a non-zero phase shift). Extensions of the existence and stability theory to the generalized NLS equation in two and higher dimensions were also developed by J.C. Saut and his co-workers.

While many mathematical results are now available for solutions of the generalized NLS equation (1.1) with non-zero boundary conditions and non-zero phase shift, dark solitons have suffered a decreasing popularity in the context of nonlinear optics. This is not only because they possess infinite energy due to non-zero boundary conditions but also because it is difficult from the experimental point of view to separate the effects of the dark soliton dynamics and the background dynamics. Also no engineering or commercial applications of dark solitons have been proposed, to the best of our knowledge.

Nevertheless, the interest in these nonlinear waveforms has recently been rejuvenated by the rapid development of a new area of physics, namely the field of Bose-Einstein condensates (BECs) [39, 40. In the latter setting, dark solitons typically move along the localized ground state trapped by the external potentials 42. The main model for BECs is a modification of the NLS equation (1.1) with an external potential, which is called the Gross-Pitaevskii (GP) equation,

$$
i u_{t}=-\frac{1}{2} u_{x x}+f\left(|u|^{2}\right) u+\epsilon V(x) u,
$$

where $\epsilon \in \mathbb{R}$ is the strength of the potential $V(x)$ and $V(x): \mathbb{R} \rightarrow \mathbb{R}$ is assumed to be a smooth function satisfying one of the three properties:

(i) $V(x)$ is bounded and decaying, e.g.

$$
\exists C>0, \kappa>0: \quad|V(x)| \leq C e^{-\kappa|x|}, \quad \forall x \in \mathbb{R}
$$

(ii) $V(x)$ is bounded but non-decaying, e.g. $V(x+d)=V(x), x \in \mathbb{R}$ with period $d>0$

(iii) $V(x)$ is unbounded, e.g. $V(x)=x^{2}+\tilde{V}(x)$, where $\tilde{V}(x)$ is bounded on $x \in \mathbb{R}$.

The last two cases are of particular interest in the context of Bose-Einstein condensates, where dynamics of dark solitons (localized dips in the nonlinear ground state trapped by the potential $V(x)$ ) was studied in many recent papers, see [5, 34 for surveys of results. Although cases (ii) and (iii) have been initially our primary motivations, this paper covers only the case (i) when $V(x)$ is bounded and exponentially 
decaying as in (1.3). In particular, we consider the class of symmetric potentials $V(-x)=V(x)$ with two examples

$$
V_{1}(x)=-\operatorname{sech}^{2}\left(\frac{\kappa x}{2}\right), \quad V_{2}(x)=x^{2} e^{-\kappa|x|}, \quad x \in \mathbb{R} .
$$

The other cases (ii) and (iii) of the potential $V(x)$ will be the subject of forthcoming studies.

We note in passing that while the potentials (1.4) are, perhaps, less customary than the standard magnetic (parabolic) and optical lattice (periodic) potentials [21, they are nonetheless still quite physically relevant. In particular, the potential $V_{1}(x)$ corresponds to a red-detuned laser beam potential, analogous to the one used in 32, 43. The potential $V_{2}(x)$ represents an all-optically trapped BEC, as modeled in [7] and experimentally implemented in [3].

The strategy of our work is to exploit solutions of the GP equation (1.2) in the limit of small strength $\epsilon$. Starting with the limit $\epsilon=0$, where both existence and stability of dark solitons with non-zero and zero speeds are known from the analysis of the NLS equation (1.1), we shall use the method of Lyapunov-Schmidt reductions and obtain existence and stability results for small $\epsilon$. From the technical point of view, we use local bifurcation analysis of solutions of the ODEs with non-zero boundary conditions similarly to [30, persistence analysis of isolated eigenvalues in the problems with small potentials similarly to [18, the count of eigenvalues in the generalized eigenvalue problem for self-adjoint operators with no spectral gaps similarly to [9], and the construction of $L^{2}$ eigenfunctions of the stability problem with fast and slow exponential decay similarly to [33. Since our starting point is the case of $\epsilon=0$, we will also give alternative rigorous proofs to the existence and stability of black solitons in the NLS equation (1.1), which complement the recent work of [31.

Our main results are listed as follows.

(i) Let $u=\phi_{0}(x-s) e^{-i \omega t+i \theta}$ be a solution of the NLS equation (1.1) with $s \in \mathbb{R}, \theta \in \mathbb{R}, \omega=f\left(q_{0}\right)$, $q_{0} \in \mathcal{I} \subset \mathbb{R}_{+}$and $\phi_{0}(x): \mathbb{R} \mapsto \mathbb{R}$ such that $\phi_{0}(x) \rightarrow \pm \sqrt{q_{0}}$ as $x \rightarrow \pm \infty$ exponentially fast. Let $s_{0}$ be the root of the function $M^{\prime}(s)$, such that

$$
M^{\prime}\left(s_{0}\right)=\int_{\mathbb{R}} V^{\prime}(x)\left[q_{0}-\phi_{0}^{2}\left(x-s_{0}\right)\right] d x=0
$$

and

$$
M^{\prime \prime}\left(s_{0}\right)=\int_{\mathbb{R}} V^{\prime \prime}(x)\left[q_{0}-\phi_{0}^{2}\left(x-s_{0}\right)\right] d x \neq 0 .
$$

Then, there exists a unique continuation of the solution $u=\phi_{\epsilon}\left(x-s_{\epsilon}\right) e^{-i \omega t+i \theta}$ of the GP equation (1.2) with $V(x)$ in (1.3) and $\epsilon$ sufficiently small, where $\phi_{\epsilon}: \mathbb{R} \mapsto \mathbb{R}$ and $\phi_{\epsilon}(x) \rightarrow \pm \sqrt{q_{0}}$ as $x \rightarrow \pm \infty$ exponentially fast, such that $\phi_{\epsilon}(x)$ and $s_{\epsilon}$ are $\epsilon$-close to $\phi_{0}(x)$ and $s_{0}$ in $L^{\infty}$-norm.

(ii) Let the solution $\phi_{0}(x)$ be spectrally stable in the time evolution of the NLS equation (1.1). Then, the solution $\phi_{\epsilon}(x)$ is spectrally unstable in the time evolution of the GP equation (1.2) for sufficiently small $\epsilon$ with exactly one real positive eigenvalue if $M^{\prime \prime}\left(s_{0}\right)<0$ and exactly two complex-conjugate eigenvalues with positive real part if $M^{\prime \prime}\left(s_{0}\right)>0$.

(iii) Let the function $u_{0}(x): \mathbb{R} \mapsto \mathbb{R}$ be close to the solution $\phi_{\epsilon}(x)$ in $L^{\infty}$-norm and satisfy the same boundary conditions $u_{0}(x) \rightarrow \pm \sqrt{q_{0}}$ as $x \rightarrow \pm \infty$. Then, the solution $u(x, t): \mathbb{R} \times \mathbb{R}_{+} \mapsto \mathbb{C}$ of the Cauchy problem to the GP equation (1.2) with $u(x, 0)=u_{0}(x)$ remains close to the dark soliton 
$\phi_{\epsilon}(x-s(t))$ with varying coordinate $s(t)$ for $0 \leq t<T$ such that $s(t)$ solves the Newton's particle equation

$$
\mu_{0} \ddot{s}-\epsilon \lambda_{0} M^{\prime \prime}(s) \dot{s}=-\epsilon M^{\prime}(s), \quad 0 \leq t<T,
$$

where $M(s)$ is the effective potential in (1.5) and (1.6), $\left(\mu_{0}, \lambda_{0}\right)$ are constants representing the soliton's mass and gain terms, and the time $T>0$ is of the order of $\mathrm{O}(1 / \epsilon)$.

Statement (i) is formulated and proved in Section 2 (see Theorem 2.12). Statement (ii) is formulated and proved in Section 3 (see Theorems 3.11] 3.14 and 3.15) under some non-degeneracy assumptions (see Corollary 3.17)and Remark 3.18). The two complex-conjugate eigenvalues with positive real part in the statement (ii) for $M^{\prime \prime}\left(s_{0}\right)>0$ follows from the linearized version of the Newton's particle equation (1.7) with $\mu_{0}>0$ and $\lambda_{0}>0$, which is rigorously derived in Section 4 (see Theorem 4.11). Constants $\left(\mu_{0}, \lambda_{0}\right)$ are defined by Remark 4.13. The linearization around a black soliton is investigated in Section 5 using numerical bifurcation theory and approximations of eigenvalues. Statement (iii) is a conjecture which is tested against appropriately crafted numerical experiments in Section 6. The summary and open problems are discussed in the concluding Section 7.

\section{Existence analysis of dark and black solitons}

We first consider the family of traveling solutions of the NLS equation (1.1). After we formulate the conditions for existence of dark and black solitons, we address persistence of stationary solutions in the Gross-Pitaevskii equation (1.2) for small $\epsilon$ and the potential $V(x)$ in (1.3). Applications of the persistence analysis to the two potentials (1.4) indicate that the families of black solitons bifurcate from the extremal points of the effective potential $M(s)$ in (1.5)-(1.6).

Definition 2.1 A dark soliton is the traveling solution of the NLS equation (1.1) in the form:

$$
u(x, t)=U(x-v t) e^{-i \omega t}, \quad U(z)=\Phi(z) e^{i \Theta(z)}, \quad z=x-v t,
$$

where $U: \mathbb{R} \mapsto \mathbb{C}, \Phi: \mathbb{R} \mapsto \mathbb{R}_{+}$, and $\Theta: \mathbb{R} \mapsto[-\pi, \pi]$ are smooth functions of their arguments, which converge exponentially fast to the boundary conditions

$$
\lim _{z \rightarrow \pm \infty} \Phi(z)=\sqrt{q_{0}}, \quad \lim _{z \rightarrow \pm \infty} \Theta(z)=\Theta_{ \pm} .
$$

Here $(\omega, v) \in \mathcal{D} \subset \mathbb{R}^{2}, q_{0} \in \mathcal{I} \subset \mathbb{R}_{+}$and $\Theta_{ \pm} \in[-\pi, \pi]$ are parameters of the solution. Moreover, the functions $\Phi(z)$ and $\Theta(z)$ can be chosen to satisfy the normalization conditions $\Phi^{\prime}(0)=0$ and $\Theta_{+}=0$. Additionally, we require that $\Phi(z)<\sqrt{q_{0}}$ on $z \in \mathbb{R}$.

Remark 2.2 The normalization conditions for $\Phi(z)$ and $\Theta(z)$ use the gauge $\left[u(x, t) \rightarrow u(x, t) e^{i \theta}\right.$, $\forall \theta \in \mathbb{R}]$ and translational $[u(x, t) \rightarrow u(x-s, t), \forall s \in \mathbb{R}]$ invariance of the NLS equation, while the linear growth of $\Theta(z)$ in $z$ is excluded by the Galileo invariance $\left[u(x, t) \rightarrow u(x-k t, t) e^{i k x-i k^{2} t / 2}, \forall k \in \mathbb{R}\right]$.

Theorem 2.3 Let $f(q)$ be $C^{1}\left(\mathbb{R}_{+}\right)$and fix $q_{0} \in \mathbb{R}_{+}$such that $f^{\prime}\left(q_{0}\right)>0$ and $c=\sqrt{q_{0} f^{\prime}\left(q_{0}\right)}>0$. The dark soliton $U(z)$ of Definition [2.1 exists if $\omega=f\left(q_{0}\right)$ and $v \in(-c, 0) \cup(0, c)$. Moreover, for these solutions, $\Phi(z)$ has a global minimum at $z=0$ with $0<\Phi(0)<\sqrt{q_{0}}$ and $\Theta(z)$ is monotonically decreasing for $v>0$ and increasing for $v<0$. 
Proof. It follows immediately that the function $U(z)$ satisfies the second-order ODE:

$$
-i v U^{\prime}+\frac{1}{2} U^{\prime \prime}+\left(\omega-f\left(|U|^{2}\right)\right) U=0,
$$

while the functions $\Phi(z)$ and $\Theta(z)$ satisfy the ODE system in the hydrodynamic form:

$$
\begin{aligned}
\Phi^{\prime \prime}-\left(\Theta^{\prime}\right)^{2} \Phi+2 v \Theta^{\prime} \Phi+2\left(\omega-f\left(\Phi^{2}\right)\right) \Phi & =0 \\
\left(\Theta^{\prime} \Phi^{2}-v \Phi^{2}\right)^{\prime} & =0
\end{aligned}
$$

Integrating the ODE (2.5) under the boundary conditions (2.2), we obtain

$$
\Theta^{\prime}=v \frac{\Phi^{2}-q_{0}}{\Phi^{2}} .
$$

As a result, the ODE (2.4) reads as follows

$$
\Phi^{\prime \prime}+2\left(\omega-f\left(\Phi^{2}\right)\right) \Phi+v^{2} \frac{\Phi^{4}-q_{0}^{2}}{\Phi^{3}}=0 .
$$

The equilibrium point $\Phi=\sqrt{q_{0}}$ exists if and only if $\omega=f\left(q_{0}\right)$ and it is a non-degenerate hyperbolic point if and only if $v^{2}<c^{2}=q_{0} f^{\prime}\left(q_{0}\right)$. Integrating the second-order ODE (2.7) subject to the boundary conditions (2.2), we obtain the quadrature

$$
\left(\Phi^{\prime}\right)^{2}-2 W\left(\Phi^{2}\right)+v^{2} \frac{\left(\Phi^{2}-q_{0}\right)^{2}}{\Phi^{2}}=0
$$

where

$$
W(q)=\int_{q_{0}}^{q}\left(f\left(q_{0}\right)-f(q)\right) d q .
$$

The non-degenerate turning point $0<\Phi_{0}<\sqrt{q_{0}}$ of the effective potential $-2 W(q)+v^{2}\left(q-q_{0}\right) / q$ exists for any $0<v^{2}<c^{2}$, such that the trajectory from the hyperbolic point turns at $\Phi(0)=\Phi_{0}$ and returns back to the hyperbolic point forming a homoclinic orbit, thus proving the statement.

Remark 2.4 It is not difficult to prove from the ODE analysis that $U(z)$ is in fact $C^{\infty}(\mathbb{R})$ for $v \in$ $(-c, 0) \cup(0, c)$ and $U(z)$ converges to the limiting values $\sqrt{q_{0}} e^{i \Theta_{ \pm}}$as $z \rightarrow \pm \infty$ at the exponential rate $\mathrm{O}\left(e^{-\sqrt{c^{2}-v^{2}} z}\right)$. Although Lemma 2.3 formulates only the sufficient condition for existence of dark solitons, it is possible to consider all other solutions of the ODE system for $\Phi(z)$ and $\Theta(z)$. The case $v^{2}>c^{2}$ corresponds to the elliptic point $\Phi=\sqrt{q_{0}}$ and has no homoclinic orbits at all. The marginal case $v^{2}=c^{2}$ may admit an algebraically decaying homoclinic orbit, which is excluded by Definition 2.1. In the case $v^{2}<c^{2}$, other homoclinic orbits can exist with $\Phi(z)>\sqrt{q_{0}}$ but they are also excluded by Definition 2.1. The only case, which may be included into the necessary and sufficient condition of existence of dark soliton, is the limit $v \rightarrow 0$. This limit leads to two different kinds of solutions which are classified in the following definition.

Definition 2.5 The limiting solution $\phi_{0}(x)=\lim _{v \downarrow 0} U(x)$ in the family of dark solitons of Definition 2.1 is said to be the black soliton if $\phi_{0}(x)$ is a real-valued smooth function on $x \in \mathbb{R}$. The black soliton is called the bubble if $\phi_{0}(-x)=\phi_{0}(x)$ with $0 \leq \phi_{0}(0)<\sqrt{q_{0}}$ and $\lim _{x \rightarrow \pm \infty} \phi_{0}(x)=\sqrt{q_{0}}$, while it is called the kink if $\phi_{0}(-x)=-\phi_{0}(x)$ with $\phi_{0}(0)=0$ and $\lim _{x \rightarrow \pm \infty} \phi_{0}(x)= \pm \sqrt{q_{0}}$, where $\phi_{0}(x)$ converges exponentially fast to the limits. 
Theorem 2.6 Let $f(q)$ satisfy the same conditions as in Lemma 2.3 and $W(q)$ be defined by (2.9). The kink solution of Definition [2.5 exists if and only if $\omega=f\left(q_{0}\right), v=0$, and $W(q)>0$ for all $0 \leq q<q_{0}$. The bubble solution of Definition 2.5 exists if and only if $\omega=f\left(q_{0}\right), v=0$, and there exists the largest value $q_{1} \in\left[0, q_{0}\right)$ that satisfies $W\left(q_{1}\right)=0$ and $W^{\prime}\left(q_{1}\right)>0$.

Proof. By Lemma 2.3, we have $0<\Phi(z)<\sqrt{q_{0}}$ and $\Theta^{\prime}(z)<0$ on $z \in \mathbb{R}$ for $v>0$ and hence no kink or bubble solutions may exist for $v>0$. Let us consider the real-valued solution $\phi_{0}(x)$ of the second-order ODE

$$
\frac{1}{2} \phi_{0}^{\prime \prime}+\left(\omega-f\left(\phi_{0}^{2}\right)\right) \phi_{0}=0
$$

The equilibrium points $\phi_{0}= \pm \sqrt{q_{0}}$ exist if and only if $\omega=f\left(q_{0}\right)$ and they are non-degenerate hyperbolic points. Integrating the second-order ODE (2.10) under the boundary conditions in Definition 2.5, we obtain

$$
\left(\phi_{0}^{\prime}\right)^{2}-2 W\left(\phi_{0}^{2}\right)=0
$$

where $W(q)$ is defined by (2.9). No turning point of $W(q)$ exists if $W(q)>0$ for any $q \in\left[0, q_{0}\right)$, and the outgoing trajectory from the hyperbolic point $\phi_{0}=\sqrt{q_{0}}$ connects the incoming trajectory to the hyperbolic point $\phi_{0}=-\sqrt{q_{0}}$ and vice versa forming a pair of heteroclinic orbits. If there exists a nondegenerate largest turning point $q_{1}$ of $W(q)$ on $q_{1} \in\left[0, q_{0}\right)$, such that $W\left(q_{1}\right)=0$ and $W^{\prime}\left(q_{1}\right)>0$, then the trajectory from the hyperbolic point $\phi_{0}=\sqrt{q_{0}}$ turns at $\phi_{0}=\sqrt{q_{1}}$ and returns back to the point $\phi_{0}=\sqrt{q_{0}}$ forming a homoclinic orbit. If the point $q_{1}$ is degenerate, such that $W\left(q_{1}\right)=W^{\prime}\left(q_{1}\right)=0$, there exists a front solution $\phi_{0}(x)$ from $\lim _{x \rightarrow-\infty} \phi_{0}(x)=\sqrt{q_{1}}$ to $\lim _{x \rightarrow \infty} \phi_{0}(x)=\sqrt{q_{0}}$ and vice versa forming a pair of fronts, which are excluded by Definition 2.5

It remains to prove that the family of dark solitons $U(x)$ for $0<v<c$ converges to the black soliton $\phi_{0}(x)$ as $v \downarrow 0$. The proof follows from the quadrature (2.8). There exist unique classical solutions $\phi_{ \pm}(z)$ on $z \in \mathbb{R}_{ \pm}$for any $v>0$ with $\Phi_{ \pm}(0)=\sqrt{q_{*}}$ found from the largest root of $-2 W\left(q_{*}\right)+v^{2}\left(q_{*}-q_{0}\right) / q_{*}=0$ on $q \in\left[0, q_{0}\right)$. In the limit $v \downarrow 0$, the root $q_{*}$ converges to 0 if $W(0)>0$ and no other roots of $W(q)$ exists on $q \in\left[0, q_{0}\right)$. Otherwise, the root $q_{*}$ converges to $q_{1}$, where $q_{1}$ is the largest root of $W(q)$ on $q \in\left[0, q_{0}\right)$. It follows from the ODE (2.6) in the limit $v \downarrow 0$ that $\Theta(z)$ is piecewise constant function on $z \in \mathbb{R}$ with a possible jump discontinuity at $z=0$.

Let us write $x=z$ for $v=0$. In the case $q_{*} \downarrow 0$ as $v \downarrow 0$, the two smooth solutions $\Phi_{ \pm}(x)$ are glued into one smooth real-valued solution $\phi_{0}(x)$ if and only if $\Theta(x)=0$ on $x \in \mathbb{R}_{+}$and $\Theta(x)=\pi$ on $x \in \mathbb{R}_{-}$(under the normalization $\Theta_{+}=0$ ). This limiting solution becomes the kink of Definition 2.5. In the case $q_{*} \downarrow q_{1}$ as $v \downarrow 0$ and $q_{1}$ is a non-degenerate root of $W(q)$, the two smooth solutions $\Phi_{ \pm}(x)$ are glued into a smooth real-valued solution $\phi_{0}(x)$ if and only if $\Theta(x)=0$ on $x \in \mathbb{R}$ (under the same normalization). This limiting solution becomes the bubble of Definition 2.5

Remark 2.7 Due to their potential stability in the time evolution of the NLS equation (1.1), only kinks are considered in the GP equation (1.2) for sufficiently small $\epsilon$. The bubbles are always unstable in the time evolution of the NLS equation (1.1) [4].

Definition 2.8 The kink mode is the stationary solution of the GP equation (1.2) in the form:

$$
u(x, t)=\phi_{\epsilon}(x) e^{-i f\left(q_{0}\right) t+i \theta}, \quad \theta \in \mathbb{R},
$$

where $\phi_{\epsilon}(x)$ is a real-valued smooth function on $x \in \mathbb{R}$, which converges exponentially fast to the boundary conditions $\lim _{x \rightarrow \pm \infty} \phi_{\epsilon}(x)= \pm \sqrt{q_{0}}$, for any $\epsilon \in \mathbb{R}$. 
Lemma 2.9 Let $\phi_{\epsilon}(x)$ be the kink mode of Definition 2.8 and $V(x)$ be the potential satisfying (1.3). Then, for any $\epsilon \neq 0$,

$$
\int_{\mathbb{R}} V^{\prime}(x)\left[q_{0}-\phi_{\epsilon}^{2}(x)\right] d x=0
$$

Proof. The stationary solutions of Definition 2.8 satisfy the second-order ODE:

$$
\frac{1}{2} \phi_{\epsilon}^{\prime \prime}+\left(f\left(q_{0}\right)-f\left(\phi_{\epsilon}^{2}\right)\right) \phi_{\epsilon}=\epsilon V(x) \phi_{\epsilon},
$$

which is generated by the Hamiltonian function

$$
E\left(\phi_{\epsilon}, \phi_{\epsilon}^{\prime}, x\right)=\frac{1}{2}\left(\phi_{\epsilon}^{\prime}\right)^{2}-W\left(\phi_{\epsilon}^{2}\right)+\frac{\epsilon}{2} V(x)\left[q_{0}-\phi_{\epsilon}^{2}\right]
$$

where $W(q)$ is given by (2.9). Therefore, the change of $E\left(\phi_{\epsilon}(x), \phi_{\epsilon}^{\prime}(x), x\right)$ at the classical solution $\phi_{\epsilon}(x)$ of the second-order ODE (2.12) is given by

$$
\frac{d E}{d x}=\epsilon V^{\prime}(x)\left[q_{0}-\phi_{\epsilon}^{2}(x)\right]
$$

Integrating this equation on $x \in \mathbb{R}$ and using the boundary conditions for $\phi_{\epsilon}(x)$ of Definition 2.8, such that $\lim _{x \rightarrow \pm \infty} E\left(\phi_{\epsilon}(x), \phi_{\epsilon}^{\prime}(x), x\right)=0$, we derive the condition (2.11).

Remark 2.10 If $V(-x)=V(x)$ and $\phi_{\epsilon}(-x)=-\phi_{\epsilon}(x)$ on $x \in \mathbb{R}$, the necessary condition (2.11) is always satisfied. The center of the kink is located at $x=0$, which is the minimal point of $V(x)$ if $V^{\prime \prime}(0)>0$ and maximal point if $V^{\prime \prime}(0)<0$.

Remark 2.11 The necessary condition (2.11) specifies restrictions on the shape of the kink mode $\phi_{\epsilon}(x)$ but does not give us any information about its existence. By using the smallness of the external parameter $\epsilon$ in front of $V(x)$, we will show that this condition is equivalent to the bifurcation equation in the Lyapunov-Schmidt reduction technique. A similar result for bright solitons was obtained in [18. A different role of the condition (2.11) was exploited in [37] in the context of local bifurcations of small gap solitons in finite periodic potentials $V(x)$.

Theorem 2.12 Let $\phi_{0}(x)$ be the kink of Definition 2.5. Let $s_{0}$ be a simple root of the function

$$
M^{\prime}(s)=\int_{\mathbb{R}} V^{\prime}(x)\left[q_{0}-\phi_{0}^{2}(x-s)\right] d x, \quad s \in \mathbb{R},
$$

such that $M^{\prime}\left(s_{0}\right)=0$ and $M^{\prime \prime}\left(s_{0}\right) \neq 0$. Let $f(q)$ be $C^{1}\left(\mathbb{R}_{+}\right)$and $V(x)$ be $C^{2}(\mathbb{R})$ satisfying (1.3). Then, there exists a unique continuation of $\phi_{0}\left(x-s_{0}\right)$ to the kink mode $\phi_{\epsilon}\left(x-s_{\epsilon}\right)$ of Definition 2.8 for sufficiently small $\epsilon$, such that $\phi_{\epsilon}(x)$ and $s_{\epsilon}$ are $\epsilon$-close to $\phi_{0}(x)$ and $s_{0}$ in the $L^{\infty}$-norm.

Proof. We use the decomposition $\phi_{\epsilon}(x)=\phi_{0}(x-s)+\varphi(x, \epsilon, s)$ and rewrite the second-order ODE (2.12) for $\phi_{\epsilon}(x)$ as the root of the nonlinear operator-valued function

$$
F(\varphi, \epsilon, s)=L_{+} \varphi+N(\varphi, s, \epsilon)+\epsilon V(x)\left[\phi_{0}(x-s)+\varphi\right]
$$


where $L_{+}: H^{2}(\mathbb{R}) \mapsto L^{2}(\mathbb{R})$ is the self-adjoint operator parameterized by $s$

$$
L_{+}=-\frac{1}{2} \partial_{x}^{2}+f\left(\phi_{0}^{2}\right)-f\left(q_{0}\right)+2 \phi_{0}^{2} f^{\prime}\left(\phi_{0}^{2}\right),
$$

and $N(\varphi, \epsilon, s): H^{2}(\mathbb{R}) \mapsto H^{2}(\mathbb{R})$ is the nonlinear vector field

$$
N=\phi_{0}\left[f\left(\left(\phi_{0}+\varphi\right)^{2}\right)-f\left(\phi_{0}^{2}\right)-2 \phi_{0} \varphi f^{\prime}\left(\phi_{0}^{2}\right)\right]+\varphi\left[f\left(\left(\phi_{0}+\varphi\right)^{2}\right)-f\left(\phi_{0}^{2}\right)\right],
$$

such that $N(\varphi, \epsilon, s)=\mathrm{o}\left(\|\varphi\|_{H^{2}}\right)$ as $\|\varphi\|_{H^{2}} \rightarrow 0$ (since $\left.f \in C^{1}\left(\mathbb{R}_{+}\right)\right)$. Because $\phi_{0}(x)$ converges to $\pm \sqrt{q_{0}}$ as $x \rightarrow \pm \infty$ exponentially fast, the essential spectrum of $L_{+}$is bounded from below by $2 c^{2}>$ 0 . The operator may have isolated positive eigenvalues and no negative eigenvalues since the kernel $L_{+} \phi^{\prime}(x-s)=0$ is a positive definite ground state. Therefore, $H^{2}(\mathbb{R})=\operatorname{Ker}\left(L_{+}\right) \oplus \operatorname{Ker}\left(L_{+}\right)^{\perp}$ and the method of Lyapunov-Schmidt reductions can be applied. Projection of $F(\varphi, \epsilon, s)$ onto $\operatorname{Ker}\left(L_{+}\right)^{\perp}$ defines a unique smooth map $(x, \epsilon, s) \mapsto \varphi \in \operatorname{Ker}\left(L_{+}\right)^{\perp} \subset H^{2}(\mathbb{R})$ such that $\|\varphi\|_{H^{2}}=\mathrm{O}(\epsilon)$ as $\epsilon \rightarrow 0$. By the Sobolev Embedded Theorem, $\|\varphi\|_{L^{\infty}}=\mathrm{O}(\epsilon)$, such that $\varphi(x)$ can be decomposed as follows:

$$
\varphi=\epsilon \varphi_{1}(x)+\tilde{\varphi}(x, \epsilon, s),
$$

where $\varphi_{1}(x)$ is specified below and $\|\tilde{\varphi}\|_{L^{\infty}}=\mathrm{o}(\epsilon)$. Projection of $F(\varphi, \epsilon, s)$ onto $\operatorname{Ker}\left(L_{+}\right)$defines the bifurcation equation:

$$
G(\epsilon, s)=\epsilon\left(\phi_{0}^{\prime}, V(x)\left(\phi_{0}+\varphi\right)\right)+\left(\phi_{0}^{\prime}, N(\varphi, \epsilon, s)\right)=\frac{\epsilon}{2} M^{\prime}(s)+\tilde{G}(\epsilon, s),
$$

where $(\cdot, \cdot)$ is the standard inner product in $L^{2}(\mathbb{R})$ and $\tilde{G}(\epsilon, s)=\mathrm{o}(\epsilon)$ as $\epsilon \rightarrow 0$. By the Implicit Function Theorem for the root of (2.15), we obtain that $s=s_{0}+\tilde{s}(\epsilon)$, where $M^{\prime}\left(s_{0}\right)=0, M^{\prime \prime}\left(s_{0}\right) \neq 0$, and $\tilde{s}=\mathrm{o}(1)$ as $\epsilon \rightarrow 0$. By the Lyapunov-Schmidt Reduction Theorem for the root of (2.14) , a unique continuation of $\phi_{0}\left(x-s_{0}\right)$ into $\phi_{\epsilon}\left(x-s_{\epsilon}\right)$ exists. In particular, the correction term $\varphi_{1}(x)$ satisfies the inhomogeneous problem

$$
L_{+} \varphi_{1}=-V(x) \phi_{0}\left(x-s_{0}\right),
$$

which has a unique solution $\varphi_{1} \in \operatorname{Ker}\left(L_{+}\right)^{\perp} \subset H^{2}(\mathbb{R})$ by the Fredholm Alternative (since $M^{\prime}\left(s_{0}\right)=0$ ).

Corollary 2.13 (i) Let $f(q)$ be $C^{2}\left(\mathbb{R}_{+}\right)$. Then, $N(\varphi, \epsilon, s)=\mathrm{O}\left(\|\varphi\|_{H^{2}}\right)$ and $\tilde{G}(\epsilon, s)=\mathrm{O}(\epsilon)$ as $\epsilon \rightarrow 0$, such that $\tilde{s}=\mathrm{O}(\epsilon)$ and $\|\tilde{\varphi}\|_{L^{\infty}}=\mathrm{O}\left(\epsilon^{2}\right)$ as $\epsilon \rightarrow 0$.

(ii) Let $f(q)$ be $C^{\infty}\left(\mathbb{R}_{+}\right)$. Then, $\tilde{s}(\epsilon)$ and $\tilde{\varphi}(x, \epsilon, s)$ are $C^{\infty}$-functions of $\epsilon$ near $\epsilon=0$.

Remark 2.14 The renormalization of $\phi^{2} \mapsto\left(\phi^{2}-q_{0}\right)$ is not needed if the potential $V(x)$ satisfies the condition (1.3). We expect that the same quantity $M(s)$ with the renormalization above can be useful to treat the other cases (ii) and (iii) of the potential term $V(x)$. However, the method of LyapunovSchmidt reductions does not work for these cases since the essential spectrum of $L_{+}$is structurally deformed if $\epsilon \neq 0$ and $V(x)$ is not decaying. In this case, the root finding problem (2.14) is not defined in $H^{2}(\mathbb{R})$ because the term $V(x) \phi_{\epsilon}(x)$ does not decay to zero exponentially fast as $|x| \rightarrow \infty$.

Example 2.15 When the cubic NLS is considered with $f(s)=s$, the value $q_{0} \in \mathbb{R}_{+}$can be normalized by $q_{0}=1$. In this case, the second-order ODE (2.3) admits an exact solution

$$
U(z)=k \tanh (k z)+i v, \quad k=\sqrt{1-v^{2}},
$$


where $v \in(-1,1)$. The other components $\Phi(z)$ and $\Theta(z)$ of the polar form (2.1) can also be evaluated explicitly. The black soliton corresponds to the kink $\phi_{0}(x)=\tanh x$. When the potential $V(x)$ is even $V(-x)=V(x)$, the function $M^{\prime}(s)$ in (2.13) can be split into two parts:

$$
M^{\prime}(s)=\int_{\mathbb{R}_{+}} V^{\prime}(x)\left[\phi_{0}^{2}(x+s)-\phi_{0}^{2}(x-s)\right] d x=L^{\prime}(s)-L^{\prime}(-s),
$$

where

$$
L(s)=\int_{\mathbb{R}_{+}} V(x)\left[q_{0}-\phi_{0}^{2}(x-s)\right] d x .
$$

If $V(x)$ is $C^{2}(\mathbb{R})$ and satisfies the decay condition $(1.3)$, the function $L(s)$ is $C^{2}(\mathbb{R})$ and $L(s) \rightarrow 0$ exponentially fast as $|s| \rightarrow \infty$. Therefore, $M^{\prime}(0)=L^{\prime}(0)-L^{\prime}(0)=0$ and one family of kink modes bifurcates from $s_{0}=0$. Additional families of kink modes of the GP equation (1.2) may bifurcate if $L(0)$ and $L^{\prime \prime}(0)$ are of the same sign. In this case, two global extrema of $L(s)+L(-s)$ exist at $s_{0}= \pm s_{*}$ with $s_{*}>0$, such that two other families of kink modes bifurcate from $s_{0}= \pm s_{*}$.

When $V=V_{1}(x), \phi_{0}=\tanh x$, and $q_{0}=1$, the function $L(s)$ is computed in the implicit form

$$
L(s)=-\int_{\mathbb{R}_{+}} \operatorname{sech}^{2}\left(\frac{\kappa x}{2}\right) \operatorname{sech}^{2}(x-s) d x .
$$

Clearly $L(0)<0$ and, as can be seen from Fig. 1(top left panel), $L^{\prime \prime}(0)>0$ for any $\kappa \neq 0$. Additionally, Fig. 1 (middle and bottom left panels) suggests that $M(s)<0$ and $M(s) \rightarrow 0$ as $s \rightarrow \infty$ for any $\kappa$. Therefore, there is only one kink mode that bifurcates from $s_{0}=0$, where $V_{1}(x)$ has a minimum.

When $V=V_{2}(x)$, the function $L(s)$ is computed in the implicit form:

$$
L(s)=\int_{\mathbb{R}_{+}} x^{2} e^{-\kappa x} \operatorname{sech}^{2}(x-s) d x,
$$

where $\kappa \in \mathbb{R}_{+}$. The above $L(s)$ can be expressed as a generalized hypergeometric function, however, we will not reproduce the resulting expression here. Instead, we note that $L(0)>0$ and

$$
L^{\prime \prime}(0)=\int_{\mathbb{R}_{+}} x^{2} e^{-\kappa x}\left[4 \operatorname{sech}^{2} x-6 \operatorname{sech}^{4} x\right] d x .
$$

When $\kappa=0, L^{\prime \prime}(0)=2$. By using the Laplace method for computations of the integrals, one can find that $L^{\prime \prime}(0)=-4 \kappa^{-3}+\mathrm{O}\left(\kappa^{-5}\right)$ as $\kappa \rightarrow \infty$. Therefore, there exists $\kappa_{0}^{ \pm} \in \mathbb{R}_{+}$with $\kappa_{0}^{-} \leq \kappa_{0}^{+}$, such that $L^{\prime \prime}(0)>0$ for $0<\kappa<\kappa_{0}^{-}$and $L^{\prime \prime}(0)<0$ for $\kappa>\kappa_{0}^{+}$. As can be seen from Fig. 1 (top right panel), $\kappa_{0}^{-}=\kappa_{0}^{+} \approx 3.21$. Additionally, Fig. 1 (middle and bottom right panels) suggest that no other extremal points of $M(s)$ exist on $s \in \mathbb{R}$ for any $\kappa \in \mathbb{R}_{+}$. When $0<\kappa<\kappa_{0}$, there exist three kink modes: two modes with $s_{0}= \pm s_{*}$ are associated with the global maxima of the effective potential $M(s)$, while the mode with $s_{0}=0$ is associated with the local minimum of $M(s)$. When $\kappa>\kappa_{0}$, no kink modes with $s_{0}= \pm s_{*}$ exist but the mode at $s_{0}=0$ corresponds to the global maximum of the effective potential $M(s)$. Hence, the structure of kink modes corresponds to a subcritical pitchfork bifurcation in the parameter $\kappa$, such that three solutions exist (at $s_{0}=0$ and $s_{0}= \pm s_{*}$ ) for $0<\kappa<\kappa_{0}$ and only one solution persists for $\kappa>\kappa_{0}$. We point out that the effective potential $M(s)$ gives a different prediction in comparison with the true potential $V_{2}(x)$ which possesses a minimum at $x=0$ and two maxima at $x= \pm 2 / \kappa$, for all $\kappa$. 

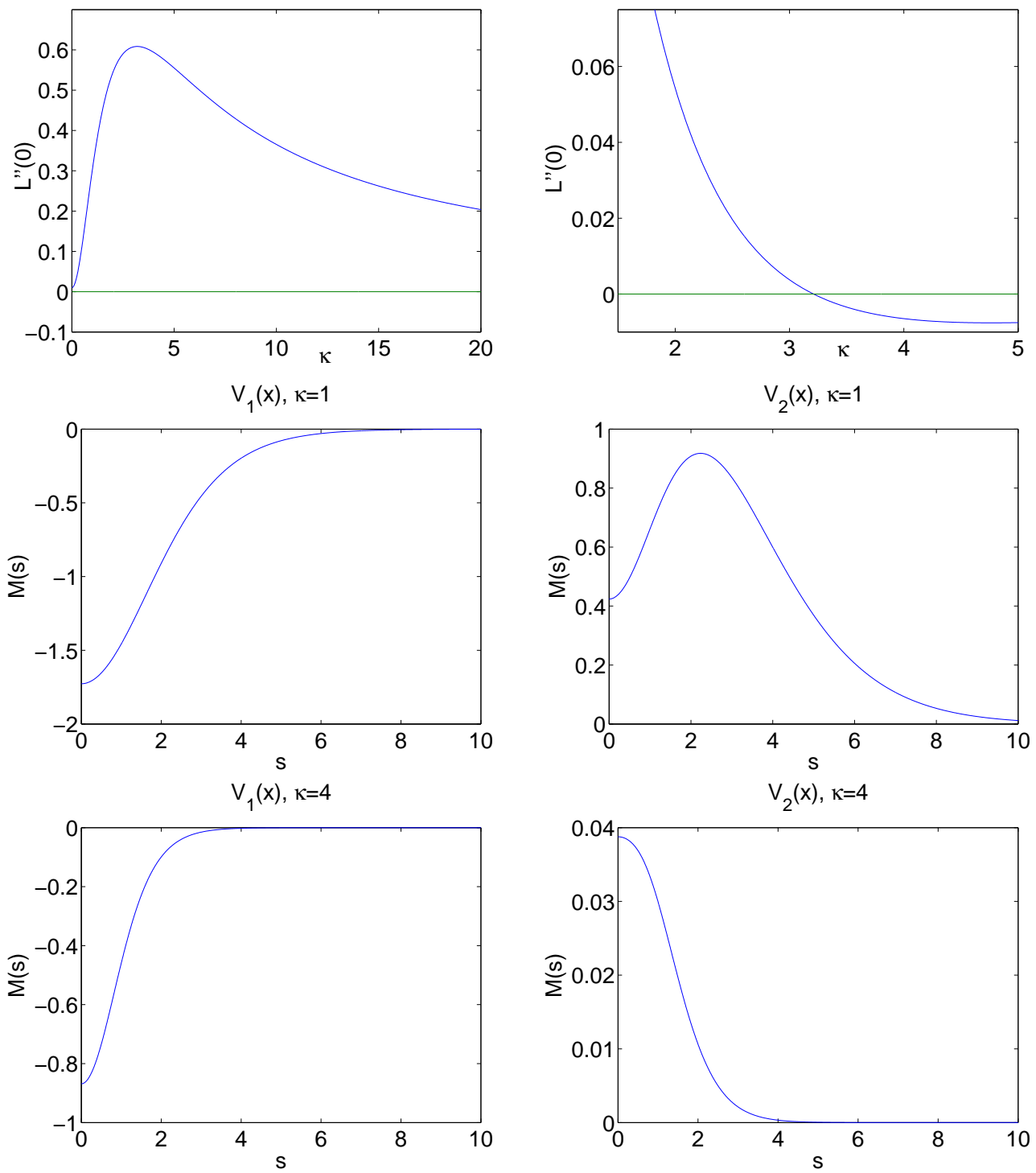

Figure 1: The effective potential evaluated numerically for $V_{1}(x)$ (left) and $V_{2}(x)$ (right): the quantity $L^{\prime \prime}(0)$ versus $\kappa$ (top panels) and the function $M(s)$ for $\kappa=1$ (middle panels) and $\kappa=4$ (bottom panels). 


\section{$3 \quad$ Stability analysis of black solitons}

We first consider the spectral stability of black solitons in the NLS equation (1.1). We will obtain conditions for spectral stability and instability of kinks and bubbles and then extend these conditions to kink modes of the GP equation (1.2). In the end of this section, we will apply these conditions to the kink modes related to the two potentials (1.4).

Definition 3.1 Let $\phi_{0}(x)$ be the black soliton of Definition 2.5. The black soliton is said to be spectrally unstable in the time evolution of the NLS equation (1.1) if there exists an eigenvector $(u, w) \in L^{2}\left(\mathbb{R}, \mathbb{C}^{2}\right)$ of the spectral problem

$$
L_{+} u=-\lambda w, \quad L_{-} w=\lambda u,
$$

for the eigenvalue $\lambda$ with $\operatorname{Re}(\lambda)>0$, where

$$
L_{+}=-\frac{1}{2} \partial_{x}^{2}+f\left(\phi_{0}^{2}\right)-f\left(q_{0}\right)+2 \phi_{0}^{2} f^{\prime}\left(\phi_{0}^{2}\right), \quad L_{-}=-\frac{1}{2} \partial_{x}^{2}+f\left(\phi_{0}^{2}\right)-f\left(q_{0}\right) .
$$

Otherwise, the black soliton is said to be spectrally stable.

Remark 3.2 The spectral problem (3.1) arises in the linearization of the NLS equation (1.1) by using the ansatz

$$
u(x, t)=e^{-i f\left(q_{0}\right) t}\left[\phi_{0}(x)+e^{\lambda t}[u(x)+i w(x)]+e^{\bar{\lambda} t}[\bar{u}(x)+i \bar{w}(x)]\right] .
$$

It will be clear from analysis of the system (3.1) that the spectral instability of black solitons is always associated with a real positive eigenvalue $\lambda$, while the spectral stability of black solitons (under a nondegeneracy constraint) corresponds to the case when the black soliton is a ground state of an equivalent variational principle. It is relatively straightforward to develop the nonlinear analysis for these two cases and to show that the spectral instability and stability of black solitons (under a non-degeneracy constraint) correspond to their orbital instability and stability. See [4, 28, 31] for developments of this nonlinear analysis.

Proposition 3.3 Let $u(x)$ be a suitable function such that $u(x)=\sqrt{q_{0}} e^{i \Theta_{ \pm}}$exponentially fast as $x \rightarrow \pm \infty$. Let the renormalized energy $E_{r}[u]$ and momentum $P_{r}[u]$ of the $N L S$ equation (1.1) be defined by

$$
\begin{aligned}
E_{r}[u] & =\frac{1}{2} \int_{\mathbb{R}}\left[\left|u_{x}\right|^{2}+2 \int_{|u|^{2}}^{q_{0}}\left(f\left(q_{0}\right)-f(q)\right) d q\right] d x, \\
P_{r}[u] & =\frac{i}{2} \int_{\mathbb{R}}\left(\bar{u} u_{x}-u \bar{u}_{x}\right)\left(1-\frac{q_{0}}{|u|^{2}}\right) d x .
\end{aligned}
$$

The family of dark solitons of Definition [2.1 is a critical point of the Lyapunov functional $\Lambda[u]=$ $E_{r}[u]+v P_{r}[u]$.

Proof. By direct differentiation, if $u=U(x)$ satisfies the second-order ODE (2.3) with $\omega=f\left(q_{0}\right)$, then the variational derivative $\left.E_{r}^{\prime}[u]\right|_{u=U}+\left.v P_{r}^{\prime}[u]\right|_{u=U}$ is zero. The second term in $P_{r}[u]$ is a Casimir 
functional, which has zero variational derivative. Therefore, the Lyapunov functional $\Lambda[u]$ is defined with accuracy to an arbitrary constant $C$ in

$$
\Lambda[u]=E_{r}[u]+v P_{r}[u]+C S[u]
$$

where

$$
S[u]=\frac{i}{2} \int_{\mathbb{R}}\left(\frac{\bar{u}_{x}}{\bar{u}}-\frac{u_{x}}{u}\right) d x=\left.[\arg (u)]\right|_{x \rightarrow-\infty} ^{x \rightarrow \infty}
$$

is the total phase shift of $u(x)$ on $x \in \mathbb{R}$ subject to the non-zero boundary conditions on $u(x)$ at infinity. In order to define the constant $C$ uniquely, we add a constraint on the variational problem by requiring that if $u=\phi e^{i \theta}, \theta^{\prime}=v\left(1-q_{0} / \phi^{2}\right)$, and $\phi=\Phi(x)$ satisfies the second-order ODE (2.7), then the first variation $\left.\tilde{\Lambda}^{\prime}[\phi]\right|_{\phi=\Phi}$ is zero, where $\tilde{\Lambda}[\phi]=\left.\Lambda[u]\right|_{u=\phi e^{i \theta}}, \theta^{\prime}=v\left(1-q_{0} / \phi^{2}\right)$. By direct differentiation, it follows immediately that $C=0$ under this requirement.

Remark 3.4 The renormalized momentum (3.4) was constructed in [23] as a difference between the standard momentum $P[u]$ associated with a solution $u(x)$ and the value $P\left[u_{0}\right]$ evaluated at the background solution $u_{0}=\sqrt{q_{0}} e^{i \operatorname{sign}(x) S_{0} / 2}$, where the value $S_{0}=S[u]$ is related to the total phase shift of the solution $u(x)$. It was shown in [1, 28] that the renormalized momentum $P_{r}[u]$ computed at the family of dark solitons $U(x)$ of Definition 2.1 defines the spectral stability and instability of dark solitons in the sense that the dark soliton is spectrally stable if $P_{r}^{\prime}(v) \geq 0$ and unstable if $P_{r}^{\prime}(v)<0$ where $P_{r}(v)=P_{r}[U]$. (The degenerate case $P_{r}^{\prime}(v)=0$ corresponds to the dark solitons which are spectrally stable and orbitally unstable. Under the non-degeneracy constraint $P_{r}^{\prime}(v) \neq 0$, the spectral stability and instability corresponds to the orbital stability and instability, see [28. In what follows, we will consider dark solitons under the non-degeneracy constraint $P_{r}^{\prime}(v) \neq 0 \forall v \in(-c, c)$.) We will show that the limit $v \downarrow 0$ is well defined and the quantity $\left.P_{r}^{\prime}\right|_{v \downarrow 0}$ determines spectral stability and instability of kinks of Definition 2.5. The latter point is missed in the recent paper [31, where stability of kinks is considered.

Lemma 3.5 Let $U(x)$ be the family of dark solitons of Definition [2.1 and $f(q)$ be $C^{2}\left(\mathbb{R}_{+}\right)$. Then, (i) the function $P_{r}(v)=P_{r}[U]$ is $C^{1}$ on $v \in(-c, 0) \cup(0, c)$ and (ii) the limiting quantity $\left.P_{r}^{\prime}\right|_{v \downarrow 0}$ is well-defined.

Proof. (i) By construction of dark solitons in Theorem [2.3, the function $P_{r}(v)=P_{r}[U]$ is represented by

$$
\begin{aligned}
P_{r}(v) & =\frac{i}{2} \int_{\mathbb{R}}\left(\bar{U} U^{\prime}-U \bar{U}^{\prime}\right)\left(1-\frac{q_{0}}{|U|^{2}}\right) d x \\
& =-v \int_{\mathbb{R}} \Phi^{2}(x)\left(1-\frac{q_{0}}{\Phi^{2}(x)}\right)^{2} d x=v N(v)+q_{0} S(v),
\end{aligned}
$$

where $N(v)$ and $S(v)$ is the total power and phase shift of the dark solitons:

$$
N(v)=\int_{\mathbb{R}}\left(q_{0}-\Phi^{2}(x)\right) d x, \quad S(v)=\int_{\mathbb{R}} \Theta^{\prime}(x) d x=\Theta_{+}-\Theta_{-} .
$$

By the ODE theory for the system (2.6) - (2.7) with $\Phi(x)>0$ on $x \in \mathbb{R}$, the map $v \mapsto(\Phi, \Theta)$ is $C^{1}$ on $v \in(-c, 0) \cup(0, c)$, such that $N(v)$ and $S(v)$ are smooth functions and so is $P_{r}(v)$. 
(ii) We will show that the functions $N(v), S(v)$ and $P_{r}(v)$ remains smooth in the limit $v \downarrow 0$. Let $U(x)$ be a dark soliton for $v \in(0, c)$ according to Definition 2.1 and $\phi_{0}(x)$ be a black soliton according to Definition 2.5. Let us consider

$$
\tilde{U}(x)=\frac{U(x)-\phi_{0}(x)}{v}=\tilde{U}_{r}(x)+i \tilde{U}_{i}(x), \quad v \in(0, c),
$$

where $\tilde{U}_{r}(x)$ and $\tilde{U}_{i}(x)$ are real-valued functions on $x \in \mathbb{R}$. By the construction of $U(x)$ and $\phi_{0}(x)$ in Theorems [2.3] and [2.6], it is clear that $\tilde{U}_{r}, \tilde{U}_{i} \in L^{\infty}(\mathbb{R})$ are continuous in $v$ for $v \in(0, c)$. We need to prove that these functions remain continuous in $v$ as $v \downarrow 0$. By separating the real and imaginary parts in the ODEs (2.3) and (2.10), we obtain the equivalent ODE system for $\tilde{U}_{r}(x)$ and $\tilde{U}_{i}(x)$ :

$$
\begin{aligned}
v \tilde{U}_{i}^{\prime}+\frac{1}{v} \phi_{0}\left(f\left(\phi_{0}^{2}\right)-f\left(|U|^{2}\right)\right)+\frac{1}{2} \tilde{U}_{r}^{\prime \prime}+\tilde{U}_{r}\left(f\left(q_{0}\right)-f\left(|U|^{2}\right)\right) & =0, \\
-\phi_{0}^{\prime}-v \tilde{U}_{r}^{\prime}+\frac{1}{2} \tilde{U}_{i}^{\prime \prime}+\tilde{U}_{i}\left(f\left(q_{0}\right)-f\left(|U|^{2}\right)\right) & =0,
\end{aligned}
$$

where $|U|^{2}=\left(\phi_{0}+v \tilde{U}_{r}\right)^{2}+v^{2} \tilde{U}_{i}^{2}$. Let us rewrite the ODE (3.10) as an inhomogeneous problem:

$$
\left(L_{-}+\tilde{L}_{-}\right) \tilde{U}_{i}=F_{-},
$$

where operator $L_{-}$is defined by (3.2) and

$$
\tilde{L}_{-}=f\left(|U|^{2}\right)-f\left(\phi_{0}^{2}\right), \quad F_{-}=-\phi_{0}^{\prime}-v \tilde{U}_{r}^{\prime} .
$$

Since $|U|^{2}(x), \phi_{0}^{2}(x)$ approach $q_{0}$ and $\tilde{U}_{r}(x), \tilde{U}_{i}(x), \phi_{0}(x)$ approach some constants exponentially fast as $|x| \rightarrow \infty$, it is clear that $\tilde{L}_{-}$is a relatively compact perturbation to $L_{-}$and $\tilde{L}_{-}, F_{-} \in L^{2}(\mathbb{R})$ for $v \in(0, c)$. By continuity of the solution $\phi_{0}(x)=\lim _{v \downarrow 0} U(x)$ in Theorem 2.6] we know that $\left\|\tilde{L}_{-}\right\|_{L^{\infty}}=\mathrm{o}(1)$ and $v\left\|\tilde{U}_{r}^{\prime}\right\|_{L^{\infty}}=\mathrm{o}(1)$ as $v \downarrow 0$.

Since $L_{-} \phi_{0}=0$ and $\phi_{0} \in L^{\infty}(\mathbb{R})$, then $\forall f \in L^{2}(\mathbb{R})$ there exists $L_{-}^{-1} f \in L^{\infty}(\mathbb{R})$ if and only if $\left(\phi_{0}, f\right)=0$. The following computation shows that this condition for $f=F_{-}-\tilde{L}_{-} \tilde{U}_{i}$ is equivalent to the ODE (3.10) and is thus satisfied on $v \in(0, c)$ :

$$
\begin{aligned}
& -\left(\phi_{0}, \phi_{0}^{\prime}\right)-v\left(\phi_{0}, \tilde{U}_{r}^{\prime}\right)+\left(\phi_{0}\left(f\left(\phi_{0}^{2}\right)-f\left(q_{0}\right), \tilde{U}_{i}\right)+\left(\phi_{0}, \tilde{U}_{i}\left(f\left(q_{0}\right)-f\left(|U|^{2}\right)\right)\right)\right. \\
& =-\left(\phi_{0}, \phi_{0}^{\prime}\right)-v\left(\phi_{0}, \tilde{U}_{r}^{\prime}\right)+\frac{1}{2}\left(\phi_{0}^{\prime \prime}, \tilde{U}_{i}\right)+\left(\phi_{0}, \tilde{U}_{i}\left(f\left(q_{0}\right)-f\left(|U|^{2}\right)\right)\right) \\
& =\left(\phi_{0},-\phi_{0}^{\prime}-v \tilde{U}_{r}^{\prime}+\frac{1}{2} \tilde{U}_{i}^{\prime \prime}+\tilde{U}_{i}\left(f\left(q_{0}\right)-f\left(|U|^{2}\right)\right)\right)=0 .
\end{aligned}
$$

Therefore, the operator $L_{-}^{-1}: L^{2}(\mathbb{R}) \mapsto L^{\infty}(\mathbb{R})$ is well-defined for the inhomogeneous problem (3.11) on $v \in(0, c)$, which can be rewritten as follows:

$$
\tilde{U}_{i}=-\left(L_{-}+\tilde{L}_{-}\right)^{-1}\left(\phi_{0}^{\prime}+v \tilde{U}_{r}^{\prime}\right)
$$

Since $\left\|\tilde{L}_{-}\right\|_{L^{\infty}}=\mathrm{o}(1)$ and $v\left\|\tilde{U}_{r}^{\prime}\right\|_{L^{\infty}}=\mathrm{o}(1)$ as $v \downarrow 0$, there exists a solution $\tilde{U}_{i} \in L^{\infty}(\mathbb{R})$ uniformly in $v \in[0, c)$, such that $\left.\tilde{U}_{i}\right|_{v \downarrow 0}=-L_{-}^{-1} \phi_{0}^{\prime} \in L^{\infty}(\mathbb{R})$. Therefore, the function $\operatorname{Im} U(x)$ is smooth as $v \downarrow 0$ and $\left.\operatorname{Im} \partial_{v} U(x)\right|_{v \downarrow 0}=\left.\tilde{U}_{i}\right|_{v \downarrow 0}=-L_{-}^{-1} \phi_{0}^{\prime}$. 
We can now use the fact that $v\left\|\tilde{U}_{i}\right\|_{L^{\infty}}=\mathrm{O}(v)$ as $v \downarrow 0$. Since $f(q)$ is $C^{2}\left(\mathbb{R}_{+}\right)$and $v\left\|\tilde{U}_{r}\right\|_{L^{\infty}}=\mathrm{o}(v)$ as $v \downarrow 0$, there exists a function $g\left(\phi_{0}, v \tilde{U}_{r}\right)$ for small $v \tilde{U}_{r}$ such that

$$
f\left(\left(\phi_{0}+v \tilde{U}_{r}\right)^{2}\right)-f\left(\phi_{0}^{2}\right)-2 v \phi_{0} \tilde{U}_{r} f^{\prime}\left(\phi_{0}^{2}\right)=v \tilde{U}_{r} g\left(\phi_{0}, v \tilde{U}_{r}\right),
$$

where $\left\|g\left(\phi_{0}, v \tilde{U}_{r}\right)\right\|_{L^{\infty}}=\mathrm{o}(v)$ as $v \downarrow 0$. Using these facts, we rewrite the ODE (3.9) as an inhomogeneous problem:

$$
\left(L_{+}+\tilde{L}_{+}\right) \tilde{U}_{r}=F_{+},
$$

where operator $L_{+}$is defined by (3.2) and

$$
\tilde{L}_{+}=f\left(|U|^{2}\right)-f\left(\phi_{0}^{2}\right)+\phi_{0} g\left(\phi_{0}, v \tilde{U}_{r}\right), \quad F_{+}=v \tilde{U}_{i}^{\prime}+\frac{1}{v} \phi_{0}\left(f\left(\left(\phi_{0}+v \tilde{U}_{r}\right)^{2}\right)-f\left(|U|^{2}\right)\right) .
$$

It is clear that $\tilde{L}_{+}, F_{+} \in L^{\infty}(\mathbb{R})$ for $v \in(0, c)$. Since $L_{+} \phi_{0}^{\prime}=0$ and $\phi_{0}^{\prime}(x) \in L^{2}(\mathbb{R})$, then $\forall f \in L^{\infty}(\mathbb{R})$ there exists $L_{+}^{-1} f \in L^{\infty}(\mathbb{R})$ if and only if $\left(\phi_{0}^{\prime}, f\right)=0$ (by the Fredholm's Alternative). The following computation shows that this condition for $f=F_{+}-\tilde{L}_{+} \tilde{U}_{r}$ is equivalent to the ODE (3.9) and is thus satisfied on $v \in(0, c)$ :

$$
\begin{aligned}
& v\left(\phi_{0}^{\prime}, \tilde{U}_{i}^{\prime}\right)+\frac{1}{v}\left(\phi_{0}^{\prime} \phi_{0},\left(f\left(\phi_{0}^{2}\right)-f\left(|U|^{2}\right)\right)\right)+\left(\phi_{0}^{\prime}\left(f\left(\phi_{0}^{2}\right)+2 \phi_{0}^{2} f^{\prime}\left(\phi_{0}^{2}\right)-f\left(|U|^{2}\right)\right), \tilde{U}_{r}\right) \\
& =v\left(\phi_{0}^{\prime}, \tilde{U}_{i}^{\prime}\right)+\frac{1}{v}\left(\phi_{0}^{\prime} \phi_{0},\left(f\left(\phi_{0}^{2}\right)-f\left(|U|^{2}\right)\right)\right)+\frac{1}{2}\left(\phi_{0}^{\prime \prime \prime}, \tilde{U}_{r}\right)+\left(\phi_{0}^{\prime}, \tilde{U}_{r}\left(f\left(q_{0}\right)-f\left(|U|^{2}\right)\right)\right) \\
& =\left(\phi_{0}^{\prime}, v \tilde{U}_{i}^{\prime}+\frac{1}{v} \phi_{0}\left(f\left(\phi_{0}^{2}\right)-f\left(|U|^{2}\right)\right)+\frac{1}{2} \tilde{U}_{r}^{\prime \prime}+\tilde{U}_{r}\left(f\left(q_{0}\right)-f\left(|U|^{2}\right)\right)\right)=0 .
\end{aligned}
$$

Therefore, the operator $L_{+}^{-1}: L^{\infty}(\mathbb{R}) \mapsto L^{\infty}(\mathbb{R})$ is well-defined for the inhomogeneous problem (3.12) on $v \in(0, c)$, which can be rewritten as follows:

$$
\tilde{U}_{r}=-\left(L_{+}+\tilde{L}_{+}\right)^{-1}\left(v \tilde{U}_{i}^{\prime}+\frac{1}{v} \phi_{0}\left(f\left(\left(\phi_{0}+v \tilde{U}_{r}\right)^{2}\right)-f\left(|U|^{2}\right)\right)\right) .
$$

Since $\left\|\tilde{L}_{+}\right\|_{L^{\infty}}=\mathrm{o}(v)$ and $v\left\|\tilde{U}_{i}\right\|_{L^{\infty}}=\mathrm{O}(v)$ as $v \downarrow 0$, there exists a solution $\tilde{U}_{r} \in L^{\infty}(\mathbb{R})$ uniformly in $v \in[0, c)$, such that $\left.\tilde{U}_{r}\right|_{v \downarrow 0}=0$ (the homogeneous solution $\phi_{0}^{\prime}(x)$ is removed from $\tilde{U}_{r}(x)$ due to the symmetry in $U(x))$. Therefore, the function $\operatorname{Re} U(x)$ is smooth as $v \downarrow 0$ and $\left.\operatorname{Re} \partial_{v} U(x)\right|_{v \downarrow 0}=\left.\tilde{U}_{r}\right|_{v \downarrow 0}=0$.

As a result, the map $v \mapsto U$ is $C^{1}$ on $v \in[0, c)$, such that $N(v)$ and $S(v)$ are smooth functions as $v \downarrow 0$ and so is $P_{r}(v)$.

Corollary 3.6 The following identities are true for $v \in(-c, 0) \cup(0, c)$

$$
P_{r}^{\prime}(v)=i \int_{\mathbb{R}}\left(U^{\prime} \partial_{v} \bar{U}-\bar{U}^{\prime} \partial_{v} U\right) d x=2 \int_{\mathbb{R}}\left(\operatorname{Re} U^{\prime} \operatorname{Im} \partial_{v} U-\operatorname{Im} U^{\prime} \operatorname{Re} \partial_{v} U\right) d x
$$

and

$$
\left.P_{r}^{\prime}\right|_{v \downarrow 0}=2\left(\phi_{0}^{\prime},\left.\operatorname{Im} \partial_{v} U\right|_{v \downarrow 0}\right)=\left.N\right|_{v \downarrow 0}+\left.q_{0} S^{\prime}\right|_{v \downarrow 0}
$$


Proof. By Lemma 3.5, the quantity $P_{r}^{\prime}(v)$ is continuous on $v \in[0, c)$. The first identity in (3.13) follows by direct differentiation:

$$
\begin{aligned}
P_{r}^{\prime}(v) & =\frac{i}{2} \int_{\mathbb{R}}\left(U^{\prime} \partial_{v} \bar{U}+\bar{U} \partial_{v} U^{\prime}-\bar{U}^{\prime} \partial_{v} U-U \partial_{v} \bar{U}^{\prime}\right) d x+\frac{i q_{0}}{2} \int_{\mathbb{R}} \partial_{v}\left(\frac{\overline{U^{\prime}}}{\bar{U}}-\frac{U^{\prime}}{U}\right) d x \\
& =i \int_{\mathbb{R}}\left(U^{\prime} \partial_{v} \bar{U}-\bar{U}^{\prime} \partial_{v} U\right) d x+\left.\frac{i}{2}\left(\bar{U} \partial_{v} U-U \partial_{v} \bar{U}\right)\right|_{x \rightarrow-\infty} ^{x \rightarrow \infty}+\left.\frac{i q_{0}}{2} \partial_{v} \log \left(\frac{\bar{U}}{U}\right)\right|_{x \rightarrow-\infty} ^{x \rightarrow \infty} \\
& =i \int_{\mathbb{R}}\left(U^{\prime} \partial_{v} \bar{U}-\bar{U}^{\prime} \partial_{v} U\right) d x
\end{aligned}
$$

Other identities follow by the substitution $U(x)=\operatorname{Re} U(x)+i \operatorname{Im} U(x)$, by the smoothness of $U(x)$ with respect to $v \in[0, c)$ and by the relation (3.7).

Example 3.7 Following Example 2.15 we consider the cubic NLS with $f(s)=s$ and $q_{0}=1$. By using the exact solution (2.17), we find for $v \in[0,1)$

$$
N(v)=2 \sqrt{1-v^{2}}, \quad S(v)=-2 \arctan \frac{\sqrt{1-v^{2}}}{v},
$$

such that

$$
P_{r}^{\prime}(v)=4 \sqrt{1-v^{2}}, \quad S^{\prime}(v)=\frac{2}{\sqrt{1-v^{2}}}
$$

and $\left.P_{r}^{\prime}\right|_{v \downarrow 0}=4,\left.S^{\prime}\right|_{v \downarrow 0}=2$.

Lemma 3.8 (i) Let $\phi_{0}(x)$ be the kink of Definition [2.5. Then, the spectrum of $L_{+}$in $L^{2}(\mathbb{R})$ consists of the positive continuous spectrum bounded away from zero by $2 c^{2}$, the kernel with the eigenfunction $\phi_{0}^{\prime}(x)$ and, possibly, a finite number of positive eigenvalues in $\left(0,2 c^{2}\right)$. The spectrum of $L_{-}$in $L^{2}(\mathbb{R})$ consists of the non-negative continuous spectrum and a single negative eigenvalue.

(ii) Let $\phi_{0}(x)$ be the bubble of Definition [2.5. Then, the spectrum of $L_{+}$in $L^{2}(\mathbb{R})$ consists of the positive continuous spectrum bounded away from zero by $2 c^{2}$, the kernel with the eigenfunction $\phi_{0}^{\prime}(x)$, a single negative eigenvalue and, possibly, a finite number of positive eigenvalues in $\left(0,2 c^{2}\right)$. The spectrum of $L_{-}$in $L^{2}(\mathbb{R})$ consists of the non-negative continuous spectrum.

Proof. Since $\phi_{0}^{2}(x) \rightarrow q_{0}$ exponentially fast as $|x| \rightarrow \infty, L_{ \pm}$in (3.2) are self-adjoint Schrödinger operators on the domain $H^{2}(\mathbb{R}) \subset L^{2}(\mathbb{R})$, which have absolutely continuous spectrum $\sigma_{c}\left(L_{ \pm}\right)$, a finite number of isolated eigenvalues of finite multiplicities $\sigma_{p}\left(L_{ \pm}\right)$, and no embedded eigenvalues or residual spectrum [14. By the Weyl's Essential Spectrum Lemma, $\sigma_{c}\left(L_{+}\right) \geq 2 q_{0} f^{\prime}\left(q_{0}\right)=2 c^{2}>0$ and $\sigma_{c}\left(L_{-}\right) \geq$ 0 , such that the continuous spectrum of $L_{+}$is bounded away from zero and the continuous spectrum of $L_{-}$touches zero. Moreover, $L_{+} \phi_{0}^{\prime}(x)=0$ and $L_{-} \phi_{0}(x)=0$ due to the translational and gauge symmetries of the NLS equation (1.1), such that $L_{+}$has a simple kernel in $L^{2}(\mathbb{R})$ while $L_{-}$has no kernel in $L^{2}(\mathbb{R})$.

(i) In the case of kinks, $\phi_{0}(x)$ has a single zero on $x \in \mathbb{R}$. By the Sturm Nodal Theorem, $\sigma_{p}\left(L_{+}\right)$ contains no negative eigenvalues and $\sigma_{p}\left(L_{-}\right)$contains exactly one negative eigenvalue.

(ii) In the case of bubbles, $\phi_{0}(x)$ has no zeros on $x \in \mathbb{R}$. By the Sturm Nodal Theorem, $\sigma_{p}\left(L_{+}\right)$ contains exactly one negative eigenvalue and $\sigma_{p}\left(L_{-}\right)$contains no negative eigenvalues. 
Lemma 3.9 Consider the constrained space

$$
X_{c}=\left\{w \in H^{2}(\mathbb{R}): \quad\left(w, \phi_{0}^{\prime}\right)=0\right\},
$$

where $\phi_{0}(x)$ is a black soliton of Definition 2.5. In the case of kinks, the operator L_ has exactly one negative eigenvalue in $X_{c}$ if $\left.P_{r}^{\prime}\right|_{v \downarrow 0}<0$ and no negative eigenvalues if $\left.P_{r}^{\prime}\right|_{v \downarrow 0}>0$, where $\left.P_{r}^{\prime}\right|_{v \downarrow 0}$ is defined by Lemma 3.5. In the case of bubbles, the operator $L_{-}$is non-negative in $X_{c}$.

Proof. We consider a constrained variational problem:

$$
\left(L_{-}-\mu\right) w=-\nu \phi_{0}^{\prime}, \quad w \in H^{2}(\mathbb{R}), \quad \mu \notin \sigma\left(L_{-}\right),
$$

where $\mu$ is the spectral parameter and $\nu$ is the Lagrange multiplier. By Lemma 3.8 there exists a unique solution $w \in H^{2}(\mathbb{R})$ for any $\mu \in\left(\mu_{0}, 0\right)$, where $\mu_{0}$ is the only negative eigenvalue of $L_{-}$in the case of kinks and $\mu_{0}=-\infty$ in the case of bubbles. By the standard variational theory (e.g. see [1, 31]), the smooth function $g(\mu)=-\left(\phi_{0}^{\prime},\left(L_{-}-\mu\right)^{-1} \phi_{0}^{\prime}\right)$ is decreasing on $\mu \in\left(\mu_{0}, 0\right)$ from $\lim _{\mu \downarrow \mu_{0}} g(\mu)=+\infty$ in the case of kinks or $\lim _{\mu \rightarrow-\infty} g(\mu)=0$ in the case of bubbles. Therefore, operator $L_{-}$is non-negative in $X_{c}$ in the case of bubbles. In the case of kinks, operator $L_{-}$is non-negative in $X_{c}$ if $\lim _{\mu \uparrow 0} g(\mu)>0$ and it has a single zero on $\mu \in\left(\mu_{0}, 0\right)$ if $\lim _{\mu \uparrow 0} g(\mu)<0$. We need to show that $\lim _{\mu \uparrow 0} g(\mu)=\left.P_{r}^{\prime}\right|_{v \downarrow 0}$.

It is clear from the solution of the variational problem (3.16) that $w \in H^{2}(\mathbb{R}) \subset L^{\infty}(\mathbb{R})$ uniformly in $\mu \in\left(\mu_{0}, 0\right]$. Since $\left(\phi_{0}, \phi_{0}^{\prime}\right)=0$, there exists a solution of the inhomogeneous problem $L_{-} w_{0}=-\phi_{0}^{\prime}$ in $w_{0} \in L^{\infty}(\mathbb{R})$ and, moreover, it follows from smoothness by Lemma 3.5 that

$$
w_{0}=\left.\tilde{U}_{i}\right|_{v \downarrow 0}=\left.\operatorname{Im} \partial_{v} U(x)\right|_{v \downarrow 0}+c \phi_{0},
$$

where $c$ is arbitrary. By the relation (3.14), we obtain $\lim _{\mu \uparrow 0} g(\mu)=\left(\phi_{0}^{\prime},\left.\operatorname{Im} \partial_{v} U\right|_{v \downarrow 0}\right)=\left.\frac{1}{2} P_{r}^{\prime}\right|_{v \downarrow 0}$.

Remark 3.10 It is interesting to note that the inhomogeneous equation $L_{-} w_{0}=-\phi_{0}^{\prime}$ admits another solution $w_{0}=x \phi_{0}(x) \notin L^{\infty}(\mathbb{R})$, which may lead to miscalculation

$$
\lim _{\mu \uparrow 0} g(\mu) \neq\left(\phi_{0}^{\prime}, x \phi_{0}\right)=\left.\frac{1}{2} N_{r}\right|_{v \downarrow 0}>0 .
$$

The linearly growing solution $x \phi_{0}(x) \notin L^{\infty}(\mathbb{R})$ is however irrelevant for the variational problem (3.16).

Theorem 3.11 (i) Let $\phi_{0}(x)$ be the kink of Definition 2.5. Then, it is spectrally stable if $\left.P_{r}^{\prime}\right|_{v \downarrow 0}>0$ and unstable if $\left.P_{r}^{\prime}\right|_{v \downarrow}<0$. In the latter case, the spectral problem (3.1) has exactly one real positive eigenvalue $\lambda$.

(ii) Let $\phi_{0}(x)$ be the bubble of Definition 2.5. Then, it is spectrally unstable with exactly one real positive eigenvalue $\lambda$ in the spectral problem (3.1).

Proof. Let $\lambda$ be a non-zero eigenvalue of the spectral problem (3.1) with $(u, w) \in H^{2}\left(\mathbb{R}, \mathbb{C}^{2}\right)$. Then, $L_{+}$ is invertible in $X_{c}$ defined by (3.15) and the component $w(x) \in X_{c}$ can be found from the generalized eigenvalue problem

$$
L_{-} w=\gamma L_{+}^{-1} w, \quad \gamma=-\lambda^{2}, \quad w \in X_{c} .
$$


Due to the equivalence above, all non-zero eigenvalues $\lambda$ of the spectral problem (3.1) can be recovered from the non-zero eigenvalues $\gamma$ of the generalized eigenvalue problem (3.17). The operators $L_{ \pm}$satisfy properties P1-P2 of the recent paper [9]. Even though $L_{-}$has no spectral gap near the origin, one can shift the generalized eigenvalue problem to the equivalent form,

$$
\left(L_{-}+\delta L_{+}^{-1}\right) w=(\gamma+\delta) L_{+}^{-1} w, \quad 0<\delta<\delta_{0},
$$

where $\delta_{0}$ is the distance from $\gamma=0$ to the first negative eigenvalue $\gamma$ if it exists or $\delta_{0}=\infty$ if not. Now

$$
\sigma_{c}\left(L_{-}+\delta L_{+}^{-1}\right) \geq \frac{\delta}{2 c^{2}}>0
$$

and the operator $\tilde{L}_{-}=L_{-}+\delta L_{+}^{-1}$ has the spectral gap near the origin.

(i) In the case of kinks, the operator $L_{+}^{-1}$ is positive in $X_{c}$. By Theorem 3 of 9 , the problem (3.18) has no eigenvalues $\gamma \in \mathbb{C}$ with $\operatorname{Im}(\gamma) \neq 0$, has no eigenvalues $\gamma \in \mathbb{R}$ such that $\left(w, L_{+}^{-1} w\right) \leq 0$ and has exactly $N=\operatorname{dim}\left(H_{L_{-}+\delta L_{+}^{-1}}^{-}\right)$eigenvalues $\gamma<0$, where $H_{L_{-}+\delta L_{+}^{-1}}^{-} \subset X_{c}$ is the invariant negative subspace of $X_{c}$ with respect to $L_{-}+\delta L_{+}^{-1}$. We will prove that $N=\operatorname{dim}\left(H_{L_{-}+\delta L_{+}^{-1}}\right)=\operatorname{dim}\left(H_{L_{-}^{-}}^{-}\right)$ for sufficiently small $\delta>0$. Indeed, continuity of isolated negative eigenvalues of $L_{-}$in $\delta$ follows by the perturbation theory since $\delta L_{+}^{-1}$ is a relatively compact perturbation to $L_{-}$in $X_{c}$. Therefore, $\operatorname{dim}\left(H_{L_{-}+\delta L_{+}^{-1}}^{-}\right) \geq \operatorname{dim}\left(H_{L_{-}}^{-}\right)$for sufficiently small $\delta>0$. Consider a splitting $X_{c}=H_{L_{-}}^{-} \oplus H_{L_{-}}^{+}$, where $H_{L_{-}}^{-}\left(H_{L_{-}}^{+}\right)$is negative (non-positive) invariant subspace of $X_{c}$ with respect to $L_{-}$, such that $\operatorname{dim}\left(H_{L_{-}}^{-}\right)<\infty$ and $\operatorname{dim}\left(H_{L_{-}}^{+}\right)=\infty$. Since $L_{+}^{-1}$ is strictly positive operator in $X_{c}$, we have

$$
\forall \delta>0, \quad \forall w \in H_{L_{-}}^{+}: \quad\left(w,\left(L_{-}+\delta L_{+}^{-1}\right) w\right) \geq \delta\left(w, L_{+}^{-1} w\right)>0 .
$$

Therefore, the operator $\left(L_{-}+\delta L_{+}^{-1}\right)$ is strictly positive on $H_{L_{-}}^{+}$and $\operatorname{dim}\left(H_{L_{-}+\delta L_{+}^{-1}}^{-}\right) \leq \operatorname{dim}\left(H_{L_{-}}^{-}\right)$. We have thus proved that $\operatorname{dim}\left(H_{L_{-}+\delta L_{+}^{-1}}^{-}\right)=\operatorname{dim}\left(H_{L_{-}}^{-}\right)$. By Lemma 3.9. $\operatorname{dim}\left(H_{L_{-}}^{-}\right)=1$ if $\left.P_{r}^{\prime}\right|_{v \downarrow 0}<0$ and $\operatorname{dim}\left(H_{L_{-}}^{-}\right)=0$ if $\left.P_{r}^{\prime}\right|_{v \downarrow 0}>0$. In the former case, $N=1$ and the kink is spectrally unstable. In the latter case, $N=0$ and the kink is spectrally stable.

(ii) In the case of bubbles, the operator $L_{-}$has no negative eigenvalues in $X_{c}$ by Lemma 3.9 . Therefore, Theorem 3 of [9] guarantees that the generalized eigenvalue problem (3.18) has no eigenvalues $\gamma \in \mathbb{C}$ with $\operatorname{Im}(\gamma) \neq 0$, has no eigenvalues $\gamma \in \mathbb{R}_{+}$such that $\left(w, L_{+}^{-1} w\right) \leq 0$ and has exactly $N=$ $\operatorname{dim}\left(H_{L_{+}^{-1}}^{-}\right)$eigenvalues $\gamma \in \mathbb{R}_{-}$with $\left(w, L_{+}^{-1} w\right) \leq 0$, where $H_{L_{+}^{-1}}^{-} \subset X_{c}$ is the invariant negative subspace of $L_{+}^{-1}$. Since all eigenvectors of $L_{+}$for non-zero eigenvalues are orthogonal to $\phi_{0}^{\prime}$ and belong to $X_{c}$, it follows immediately that $N=\operatorname{dim}\left(H_{L_{+}^{-1}}^{-}\right)=1$.

Remark 3.12 The statement (i) of Theorem 3.11 extends the stability-instability theorem in 28 from dark solitons with $v \neq 0$ to kinks with $v=0$. This connection is missed in the recent paper 31. where the same result was obtained by the Vakhitov-Kolokolov method (used in [1]) and the variational principle (used in [4]) without the proof that $\lim _{\mu \uparrow 0} g(\mu)=\left.\frac{1}{2} P_{r}^{\prime}\right|_{v \downarrow 0}$.

The statement (ii) of Theorem 3.11 was proved differently in [4] by using a variational technique. We note that $P_{r}(v)<0$ for $v \in(0, c)$ and $\lim _{v \downarrow 0} P_{r}(v)=0$ in the case of bubbles of Definition 2.5. Therefore, if $\left.P_{r}^{\prime}\right|_{v \downarrow 0} \neq 0$, then $\left.P_{r}^{\prime}\right|_{v \downarrow 0}<0$, such that the statement (i) for kinks extends formally to the statement (ii) for bubbles. 
Definition 3.13 Let $\phi_{\epsilon}(x)$ be the kink mode of Definition 2.8. The kink mode is said to be spectrally unstable in the time evolution of the GP equation (1.2) if there exists an eigenvector $(u, w) \in L^{2}\left(\mathbb{R}, \mathbb{C}^{2}\right)$ of the spectral problem

$$
\mathcal{L}_{+} u=-\lambda w, \quad \mathcal{L}_{-} w=\lambda u,
$$

for the eigenvalue $\lambda$ with $\operatorname{Re}(\lambda)>0$, where

$$
\mathcal{L}_{+}=-\frac{1}{2} \partial_{x}^{2}+f\left(\phi_{\epsilon}^{2}\right)-f\left(q_{0}\right)+2 \phi_{\epsilon}^{2} f^{\prime}\left(\phi_{\epsilon}^{2}\right)+\epsilon V(x), \quad \mathcal{L}_{-}=-\frac{1}{2} \partial_{x}^{2}+f\left(\phi_{\epsilon}^{2}\right)-f\left(q_{0}\right)+\epsilon V(x) .
$$

Otherwise, the kink mode is said to be spectrally stable.

Theorem 3.14 Let $\phi_{\epsilon}(x)$ be the kink mode of Definition [2.8. Assume that the operators $\mathcal{L}_{ \pm}$have $n_{ \pm}$negative eigenvalues and empty kernels in $L^{2}(\mathbb{R})$. Assume that all embedded (purely imaginary) eigenvalues of the spectral problem (3.19) are algebraically simple. Then, the spectral problem (3.19) for $(u, w) \in L^{2}\left(\mathbb{R}, \mathbb{C}^{2}\right)$ has exactly $N_{c}$ complex eigenvalues $\lambda$ in the first quadrant, $N_{i}^{-}$purely imaginary eigenvalues $\lambda$ with $\operatorname{Im}(\lambda)>0$ and $\left(w, \mathcal{L}_{+}^{-1} w\right) \leq 0$, and $N_{r}=N_{r}^{+}+N_{r}^{-}$real positive eigenvalues $\lambda$, where $N_{r}^{+}$corresponds to eigenvalues with $\left(w, \mathcal{L}_{+}^{-1} w\right) \leq 0$ and $N_{r}^{-}$corresponds to eigenvalues with $\left(w, \mathcal{L}_{+}^{-1} w\right) \geq 0$, such that

$$
N_{r}^{+}+N_{i}^{-}+N_{c}=n_{+}, \quad N_{r}^{-}+N_{i}^{-}+N_{c}=n_{-},
$$

where multiple eigenvalues are accounted up to their algebraic multiplicities.

Proof. Since $V(x) \rightarrow 0$ and $\phi_{\epsilon}^{2}(x) \rightarrow q_{0}$ exponentially fast as $|x| \rightarrow \infty$, operators $\mathcal{L}_{ \pm}$have the absolutely continuous spectrum such that $\sigma_{c}\left(\mathcal{L}_{+}\right) \geq 2 c^{2}>0$ and $\sigma_{c}\left(\mathcal{L}_{-}\right) \geq 0$. Since the kernel of $\mathcal{L}_{+}$is empty in $L^{2}(\mathbb{R})$ by assumption, the generalized eigenvalue problem (3.17) for operators $\mathcal{L}_{ \pm}$is rewritten in the unconstrained space:

$$
\mathcal{L}_{-} w=\gamma \mathcal{L}_{+}^{-1} w, \quad \gamma=-\lambda^{2}, \quad w \in H^{2}(\mathbb{R}) .
$$

The kernel of $\mathcal{L}_{-}$is empty in $L^{2}(\mathbb{R})$ since $\mathcal{L}_{-} \phi_{\epsilon}=0$ and $\phi_{\epsilon} \in L^{\infty}(\mathbb{R}), \phi_{\epsilon} \notin L^{2}(\mathbb{R})$. The generalized eigenvalue problem (3.22) can be rewritten in the equivalent form,

$$
\left(\mathcal{L}_{-}+\delta \mathcal{L}_{+}^{-1}\right) w=(\gamma+\delta) \mathcal{L}_{+}^{-1} w
$$

where $\delta>0$ is sufficiently small. Properties P1-P2 of [9] are satisfied and Theorem 3 of [9] gives the relations

$$
N_{r}^{+}+N_{i}^{-}+N_{c}=\operatorname{dim}\left(H_{\mathcal{L}_{+}^{-1}}^{-}\right), \quad N_{r}^{-}+N_{i}^{-}+N_{c}=\operatorname{dim}\left(H_{\mathcal{L}_{-}+\delta \mathcal{L}_{+}^{-1}}\right)
$$

for sufficiently small $\delta>0$, where $H_{\mathcal{L}_{+}^{-1}}^{-}$and $H_{\mathcal{L}_{-}+\delta \mathcal{L}_{+}^{-1}}^{-}$are invariant negative subspaces of $L^{2}(\mathbb{R})$ with respect to $\mathcal{L}_{+}^{-1}$ and $\mathcal{L}_{-}+\delta \mathcal{L}_{+}^{-1}$ respectively. It follows immediately that $\operatorname{dim}\left(H_{\mathcal{L}_{+}^{-1}}^{-}\right)=n_{+}$. By continuity of eigenvalues and the relative compactness of $\mathcal{L}_{+}^{-1}$ with respect to $\mathcal{L}_{-}$, it follows that $\operatorname{dim}\left(H_{\mathcal{L}_{-}}^{-}\right) \leq$ $\operatorname{dim}\left(H_{\mathcal{L}_{-}+\delta \mathcal{L}_{+}^{-1}}^{-}\right)$. We shall prove that $\operatorname{dim}\left(H_{\mathcal{L}_{-}+\delta \mathcal{L}_{+}^{-1}}^{-}\right)=\operatorname{dim}\left(H_{\mathcal{L}_{-}}^{-}\right)$. The operator $\mathcal{L}_{-}+\delta \mathcal{L}_{+}^{-1}$ may have additional negative eigenvalues compared to operator $\mathcal{L}_{-}$if and only if some eigenvalues bifurcate as $\delta \neq 0$ from the end point of the continuous spectrum of $\mathcal{L}_{-}$by means of the edge bifurcation [11, 20, 45]. 
In order to analyze the edge bifurcation, we rewrite the eigenvalue problem $\left(\mathcal{L}_{-}+\delta \mathcal{L}_{+}^{-1}\right) w=\mu w$ in the equivalent form:

$$
(\mathcal{L}+\delta \mathcal{M}) w=\mu w, \quad w \in L^{2}(\mathbb{R})
$$

where

$$
\mathcal{L}=\mathcal{L}_{-}+\delta\left(2 c^{2}-\frac{1}{2} \partial_{x}^{2}\right)^{-1}, \quad \mathcal{M}=\mathcal{L}_{+}^{-1}\left(f\left(\phi_{\epsilon}^{2}\right)-f\left(q_{0}\right)+2 \phi_{\epsilon}^{2} f^{\prime}\left(\phi_{\epsilon}^{2}\right)+\epsilon V(x)\right)\left(2 c^{2}-\frac{1}{2} \partial_{x}^{2}\right)^{-1}
$$

where $\mathcal{M}$ is a relatively compact perturbation to the unbounded operator $\mathcal{L}$. The continuous spectrum of $\mathcal{L}$ is bounded from below by $\sigma_{c}(\mathcal{L}) \geq \frac{\delta}{2 c^{2}}$. By the theory of edge bifurcations (see review in [20]), the new eigenvalue $\mu=\mu_{\delta}$, if it bifurcates from the end point of $\sigma_{c}(\mathcal{L})$, has the expansion $\mu_{\delta}=$ $\frac{\delta}{2 c^{2}}-\alpha \delta^{2}+\mathrm{O}\left(\delta^{3}\right)$, where $\alpha$ is positive constant. Therefore, there exists sufficiently small $\delta>0$, such that $\mu_{\delta}>0$. As a result, the edge bifurcation does not change the number of negative eigenvalues of $\mathcal{L}_{-}+\delta \mathcal{L}_{+}^{-1}$ compared to $\mathcal{L}_{-}$and $\operatorname{dim}\left(H_{\mathcal{L}_{-}+\delta \mathcal{L}_{+}^{-1}}\right)=\operatorname{dim}\left(H_{\mathcal{L}_{-}}^{-}\right)=n_{-}$.

Theorem 3.15 Let $\phi_{0}(x)$ be the kink of Definition 2.5 and $M^{\prime}(s)$ be defined by (2.13). Let $\phi_{\epsilon}(x)$ be a unique continuation of the kink $\phi_{0}\left(x-s_{0}\right)$ in Theorem 2.12 from the root $s_{0}$ such that $M^{\prime}\left(s_{0}\right)=0$ and $M^{\prime \prime}\left(s_{0}\right) \neq 0$. Then, the operators $\mathcal{L}_{ \pm}$have $n_{ \pm}$negative eigenvalues and empty kernels in $L^{2}(\mathbb{R})$ for sufficiently small $\epsilon$ with $n_{+}=1, n_{-}=1$ for $M^{\prime \prime}\left(s_{0}\right)>0$ and $n_{+}=0, n_{-}=1$ for $M^{\prime \prime}\left(s_{0}\right)<0$.

Proof. It follows by Theorem 2.12 that $\phi_{\epsilon}=\phi_{0}(x-s)+\epsilon \varphi_{1}(x)+\tilde{\varphi}(x, \epsilon, s)$ and $s=s_{0}+\tilde{s}(\epsilon)$, where $\|\tilde{\varphi}\|_{L^{\infty}}=\mathrm{o}(\epsilon)$ and $|\tilde{s}|=\mathrm{o}(1)$ as $\epsilon \rightarrow 0$. Therefore, operators $\mathcal{L}_{ \pm}$in (3.20) are represented by

$$
\mathcal{L}_{ \pm}=L_{ \pm}+\epsilon M_{ \pm}+\tilde{M}_{ \pm}
$$

where $L_{ \pm}$are given by (3.2), $M_{ \pm}$are given by

$$
M_{+}=V(x)+6 \phi_{0} \varphi_{1} f\left(\phi_{0}^{2}\right)+4 \phi_{0}^{3} \varphi_{1} f^{\prime \prime}\left(\phi_{0}^{2}\right), \quad M_{-}=V(x)+2 \phi_{0} \varphi_{1} f\left(\phi_{0}^{2}\right),
$$

and $\left\|\tilde{M}_{ \pm}\right\|_{L^{\infty}}=\mathrm{o}(\epsilon)$ as $\epsilon \rightarrow 0$. We note that $\epsilon M_{+}+\tilde{M}_{+}=\epsilon V(x)+D_{\varphi} N(\varphi, s, \epsilon)$, where $D_{\varphi} N$ is the Jacobian of the nonlinear function in (2.14). By using the inhomogeneous equation (2.16) for the correction term $\varphi_{1}(x)$, we compute

$$
\left(\phi_{0}^{\prime}\left(x-s_{0}\right), M_{+} \phi_{0}^{\prime}\left(x-s_{0}\right)\right)=-\left(\phi_{0}^{\prime}, V^{\prime} \phi_{0}\right)-\left(\phi_{0}^{\prime}, L_{+} \varphi_{1}^{\prime}\right)=-\frac{1}{2} M^{\prime \prime}\left(s_{0}\right) .
$$

By the regular perturbation theory, the zero eigenvalue of $L_{+}$becomes a non-zero eigenvalue $\lambda_{\epsilon}$ of $\mathcal{L}_{+}$ for small $\epsilon$, such that

$$
\lambda_{\epsilon}=\epsilon \lambda_{1}+\tilde{\lambda}, \quad \lambda_{1}=-\frac{M^{\prime \prime}\left(s_{0}\right)}{2\left\|\phi_{0}^{\prime}\right\|_{L^{2}}^{2}},
$$

where $\tilde{\lambda}=\mathrm{o}(\epsilon)$ as $\epsilon \rightarrow 0$. Since the zero eigenvalue of $L_{+}$is simple and positive eigenvalues of $L_{+}$are bounded away from zero, the kernel of $\mathcal{L}_{+}$is empty, such that $n_{+}=1$ for $M^{\prime \prime}\left(s_{0}\right)>0$ and $n_{+}=0$ for $M^{\prime \prime}\left(s_{0}\right)<0$ for sufficiently small $\epsilon$. By the Implicit Function Theorem applied to $\phi_{\epsilon}(x)$ (since $\phi_{0}(x)$ has only one simple zero at $x=0$, the function $\phi_{\epsilon}(x)$ has only one node on $x \in \mathbb{R}$ for sufficiently small $\epsilon$. We recall that $\mathcal{L}_{-} \phi_{\epsilon}=0$ and $\phi_{\epsilon} \in L^{\infty}(\mathbb{R}), \phi_{\epsilon} \notin L^{2}(\mathbb{R})$. By the Sturm Nodal Theorem, the kernel of $\mathcal{L}_{-}$is empty and $n_{-}=1$ for sufficiently small $\epsilon$. 
Remark 3.16 Let $f \in C^{2}\left(\mathbb{R}_{+}\right)$. Then $\left\|\tilde{M}_{ \pm}\right\|_{L^{\infty}}=\mathrm{O}\left(\epsilon^{2}\right)$ and $\tilde{\lambda}=\mathrm{O}\left(\epsilon^{2}\right)$ as $\epsilon \rightarrow 0$.

Corollary 3.17 The kink mode with $M^{\prime \prime}\left(s_{0}\right)<0$ is spectrally unstable with exactly one real positive eigenvalue $\lambda$ in the spectral problem (3.19) for sufficiently small $\epsilon$. The kink mode with $M^{\prime \prime}\left(s_{0}\right)>0$ may have up to two unstable eigenvalues $\lambda$ in the spectral problem (3.19).

Proof. If $M^{\prime \prime}\left(s_{0}\right)<0$, then $n_{+}=0, n_{-}=1$ and the count of eigenvalues (3.21) gives $N_{r}^{+}=N_{i}^{-}=$ $N_{c}=0$ and $N_{r}^{-}=1$. If $M^{\prime \prime}\left(s_{0}\right)>0$, then $n_{+}=n_{-}=1$ and the count of eigenvalues may give either $N_{i}^{-}+N_{c}=1, N_{r}^{+}=N_{r}^{-}=0$ or $N_{i}^{-}=N_{c}=0, N_{r}^{+}=N_{r}^{-}=1$. In the cases $N_{c}=1$ or $N_{r}^{+}=N_{r}^{-}=1$, there are two unstable and no embedded eigenvalues in the spectral problem (3.19). In the case $N_{i}^{-}=1$, the pair of embedded eigenvalues is simple, such that the last assumption of Theorem 3.14 is satisfied.

Remark 3.18 Asymptotic approximations of eigenvalues $\lambda$ and precise statements on unstable eigenvalues in the case $M^{\prime \prime}\left(s_{0}\right)>0$ are obtained in Section 4 under non-degeneracy assumptions $\left.P_{r}^{\prime}\right|_{v \downarrow 0} \neq 0$ and $\left.S^{\prime}\right|_{v \downarrow 0} \neq 0$. By Corollary 4.12 the case $N_{i}^{-}=N_{c}=0, N_{r}^{+}=N_{r}^{-}=1$ occurs for $\left.P_{r}^{\prime}\right|_{v \downarrow 0}<0$ and the case $N_{r}^{+}=N_{r}^{-}=N_{i}^{-}=0, N_{c}=1$ occurs for $\left.P_{r}^{\prime}\right|_{v \downarrow 0}>0$.

Example 3.19 Continuing Examples 2.15 and 3.7 we consider the cubic NLS equation with $f(s)=s$, $q_{0}=1$ and $\left.P_{r}^{\prime}\right|_{v \downarrow 0}=4>0$. When the potential $V(x)$ is even with $V(-x)=V(x)$, the function $M^{\prime}(s)$ is represented by $M^{\prime}(s)=L^{\prime}(s)-L^{\prime}(-s)$, where $L(s)$ is given by (2.18). One family of kink modes with $s_{0}=0$ always bifurcates with $M^{\prime \prime}(0)=2 L^{\prime \prime}(0)$.

When $V=V_{1}(x)$, it follows from Fig. 1 (top left panel) that $L^{\prime \prime}(0)>0$ for any $\kappa \neq 0$. The kink mode with $s_{0}=0$ corresponds to the minimum of $M(s)$ and it is unstable with two complex conjugate eigenvalues, according to Remark 3.18 .

When $V=V_{2}(x)$, it follows from Fig. 1 (top right panel) that there exists $0<\kappa_{0}<\infty$ such that $L^{\prime \prime}(0)>0$ for $0<\kappa<\kappa_{0}$ and $L^{\prime \prime}(0)<0$ for $\kappa>\kappa_{0}$. For $0<\kappa<\kappa_{0}$, a pair of kink modes bifurcates from $s_{0}= \pm s_{*}$ with $M^{\prime \prime}\left(s_{0}\right)<0$. These modes correspond to the maxima of the effective potential $M(s)$ and they are unstable with one real eigenvalue, according to Corollary [3.17] The kink mode with $s_{0}=0$ corresponds to the minimum of the effective potential $M(s)$ and it is unstable with two complex eigenvalues for $0<\kappa<\kappa_{0}$. On the other hand, it corresponds to the maximum of $M(s)$ and it is unstable with a simple real positive eigenvalue for $\kappa>\kappa_{0}$. This scenario indicates the subcritical pitchfork bifurcation at $\kappa=\kappa_{0}$. We will illustrate this bifurcation in Section 5 .

\section{Eigenfunctions and eigenvalues of kinks}

We report here asymptotic analysis of the spectral problem (3.19) in the limit of small $\lambda$ and $\epsilon$. This asymptotic analysis is needed to complete the stability analysis of kink modes with $M^{\prime \prime}\left(s_{0}\right)>0$ which is not conclusive in Corollary 3.17. We will show that if the kink is stable in the linear problem (3.1) for $\epsilon=0$, then the pair of zero eigenvalues of the spectral problem (3.19) at $\epsilon=0$ splits into a pair of purely imaginary eigenvalues at $\mathrm{O}(\epsilon)$ and bifurcates into a quartet of four complex eigenvalues (two of which are unstable) at $\mathrm{O}\left(\epsilon^{3 / 2}\right)$. These eigenvalues $\lambda$ for small $\epsilon$ correspond to the eigenvectors $(u, w) \in L^{2}\left(\mathbb{R}, \mathbb{C}^{2}\right)$, persistence of which in $\epsilon$ follows by Theorem 3.14 . 
From a technical point of view, our analysis is complicated by the fact that the eigenvalues $\lambda \in i \mathbb{R}$ are embedded into the continuous spectrum of the non-self-adjoint problem (3.19). Since we are interested in specific information about bifurcations of eigenvalues near the point $\lambda=0$, we have to abandon the generalized eigenvalue problem (3.22) and to work directly with the non-self-adjoint eigenvalue problem (3.19).

One way to deal with this problem is to introduce exponential weights which move branches of the continuous spectrum from the imaginary axis (see [38 and references therein). However, there are two branches of the continuous spectrum, and, independently of the weight parameter, one branch moves to the left and the other branch moves to the right of the imaginary axis. Any eigenvalues that bifurcate off the imaginary axis may become resonant poles when the weight parameter is sent to zero unless specific information about the decay rate of eigenfunctions is available. However, if this information were available, one could avoid the technique of exponential weights and perform a direct analysis of the eigenfunctions and eigenvalues of the problem (3.19).

Another way to deal with this problem is to consider the Evans function with careful analysis of fast and slow decaying solutions (see [19] and reference therein). By using the Gap Lemma, the Evans function can be appropriately extended across the continuous spectrum with a full account of the branch points on the imaginary axis. Information about small eigenvalues is drawn from the derivatives of the Evans function with respect to $\lambda$ and $\epsilon$ near $\lambda=0$ and $\epsilon=0$. However, computational formulas become more and more involved when higher-order derivatives of the Evans function are needed and this has caused some miscalculations in the past (see Appendix A in [4]]).

Our treatment of the problem brings together the analysis of fast and slow decaying solutions in the two approaches above. To avoid complications, it is based on direct analysis of eigenfunctions and eigenvalues of the spectral problem (3.19) expanded in powers of $\epsilon^{1 / 2}$. We will obtain a characteristic equation for small eigenvalue $\lambda$ versus small parameter $\epsilon$.

Lemma 4.1 Let $\phi_{0}(x)$ be the kink of Definition 2.5 and $U(x)$ be the dark soliton of Definition 2.1] for $v>0$. Let operators $L_{ \pm}$be defined by (3.2). The uncoupled homogeneous problems

$$
L_{+} u_{0}=0, \quad L_{-} w_{0}=0
$$

admit four linearly independent solutions:

(i) exponentially decaying eigenfunction $u_{0}=\phi_{0}^{\prime}(x)$ in $L^{2}(\mathbb{R})$

(ii) bounded eigenfunction $w_{0}=\phi_{0}(x)$ in $L^{\infty}(\mathbb{R})$

(iii) unbounded linearly growing solution $w_{0}=x \phi_{0}(x)-\left.\operatorname{Im} \partial_{v} U(x)\right|_{v \downarrow 0}$

(iv) and an unbounded exponentially growing solution $u_{0}$

The uncoupled inhomogeneous problems

$$
L_{+} u_{1}=-w_{0}, \quad L_{-} w_{1}=u_{0}
$$

admit solutions in the same order:

(i) bounded eigenfunction $w_{1}=-\left.\operatorname{Im} \partial_{v} U(x)\right|_{v \downarrow 0}$ in $L^{\infty}(\mathbb{R})$ 
(ii) a bounded eigenfunction $u_{1}$ in $L^{\infty}(\mathbb{R})$

(iii) an unbounded exponentially growing solution $u_{1}$ if $\left.S^{\prime}\right|_{v \downarrow 0} \neq 0$

(iv) and an unbounded exponentially growing solution $w_{1}$

The uncoupled inhomogeneous problems

$$
L_{+} u_{2}=-w_{1}, \quad L_{-} w_{2}=u_{1}
$$

admit no solutions in $L^{\infty}(\mathbb{R})$ if $\left.P_{r}^{\prime}\right|_{v \downarrow 0} \neq 0$.

Proof. It follows from the proofs of Lemmas 3.8 and 3.9 that

$$
L_{+} \phi_{0}^{\prime}=0, \quad L_{-} \phi_{0}=0, \quad L_{-} x \phi_{0}=-\phi_{0}^{\prime},\left.\quad L_{-} \operatorname{Im} \partial_{v} U(x)\right|_{v \downarrow 0}=-\phi_{0}^{\prime},
$$

which proves (i)-(iii) for $u_{0}$ and $w_{0}$ and (i) for $w_{1}$. Existence of an exponentially growing solution $u_{0}$ in (iv) follows from the fact that the Wronskian determinant of two linearly independent solutions of $L_{+} u_{0}=0$ is constant in $x$. Existence of bounded solution $u_{1}$ in (ii) follows from the fact that $\left(\phi_{0}^{\prime}, \phi_{0}\right)=0$, such that $L_{+}^{-1} \phi_{0} \in L^{\infty}(\mathbb{R})$. The solution $u_{1}$ in (iii) grows exponentially since the Fredholm Alternative is not satisfied for $w_{0}$ in (iii):

$$
\left(\phi_{0}^{\prime}, x \phi_{0}(x)-\left.\operatorname{Im}_{v} U(x)\right|_{v \downarrow 0}\right)=\left.\frac{1}{2} N\right|_{v \downarrow 0}-\left.\frac{1}{2} P_{r}^{\prime}\right|_{v \downarrow 0}=-\left.\frac{q_{0}}{2} S^{\prime}\right|_{v \downarrow 0} \neq 0,
$$

where the relation (3.14) has been used. Existence of an exponentially growing solution $w_{1}$ in (iv) follows from the fact that $L_{-}^{-1} u_{0}$ has the same exponential growth in $x$ as $u_{0}$ in (iv). The solution $u_{2}$ of $L_{+} u_{2}=\left.\operatorname{Im} \partial_{v} U(x)\right|_{v \downarrow 0}$ grows exponentially since the Fredholm Alternative is not satisfied:

$$
\left(\phi_{0}^{\prime},\left.\operatorname{Im} \partial_{v} U(x)\right|_{v \downarrow 0}\right)=\left.\frac{1}{2} P_{r}^{\prime}\right|_{v \downarrow 0} \neq 0
$$

The solution $w_{2}$ of $L_{-} w_{2}=u_{1}$ in (ii) grows linearly due to the same reason since

$$
\left(\phi_{0}, u_{1}\right)=-\left(L_{+} u_{1}, u_{1}\right) \neq 0 .
$$

The last inequality is due to the non-negativity of $L_{+}$for kinks and the orthogonality of the odd function $u_{1}$ to the even function $\phi_{0}^{\prime}$ of the kernel of $L_{+}$.

Definition 4.2 Let $\lambda$ be fixed in the strip $\left\{\lambda \in \mathbb{C}: 0<\operatorname{Re} \lambda<c^{2}\right\}$ and define $\kappa_{ \pm}(\lambda)$ from the roots of the characteristic equations

$$
\operatorname{Re} \kappa_{ \pm}>0: \quad \kappa_{ \pm}^{2}=2 c^{2}\left(1 \pm \sqrt{1-\frac{\lambda^{2}}{c^{4}}}\right)
$$

such that $\kappa_{+} \kappa_{-}=2 \lambda$ and $\kappa_{+}=\sqrt{4 c^{2}-\kappa_{-}^{2}}$.

Remark 4.3 The roots $\kappa_{ \pm}$can be expanded in the Taylor series near $\lambda=0$, such that

$$
\kappa_{+}(\lambda)=2 c\left(1-\frac{\lambda^{2}}{8 c^{4}}+\mathrm{O}\left(\lambda^{4}\right)\right), \quad \kappa_{-}(\lambda)=\frac{\lambda}{c}\left(1+\frac{\lambda^{2}}{8 c^{4}}+\mathrm{O}\left(\lambda^{4}\right)\right) .
$$


Lemma 4.4 Let $\phi_{\epsilon}(x)$ be the kink mode of Definition 2.8 in the domain $-\epsilon_{0}<\epsilon<\epsilon_{0}$ for some $\epsilon_{0}>0$. There exist four fundamental solutions $(u, w)$ of the spectral problem (3.19) for any $\lambda$ in the strip $\left\{\lambda \in \mathbb{C}: 0<\operatorname{Re} \lambda<c^{2}\right\}$, such that

$$
\left(\begin{array}{c}
u \\
w
\end{array}\right)_{ \pm} \rightarrow\left(\begin{array}{c}
\kappa_{ \pm} \\
-\kappa_{\mp}
\end{array}\right) e^{\kappa_{ \pm} x} \quad \text { as } \quad x \rightarrow-\infty
$$

and

$$
\left(\begin{array}{c}
\tilde{u} \\
\tilde{w}
\end{array}\right)_{ \pm} \rightarrow\left(\begin{array}{c}
\kappa_{ \pm} \\
-\kappa_{\mp}
\end{array}\right) e^{-\kappa_{ \pm} x} \quad \text { as } \quad x \rightarrow+\infty
$$

Proof. For any $\lambda$ in the strip $\left\{\lambda \in \mathbb{C}: 0<\operatorname{Re} \lambda<c^{2}\right\}$, the two roots $\kappa_{+}(\lambda)$ and $\kappa_{-}(\lambda)$ of the characteristic equations (4.3) are distinct and $R \kappa_{ \pm}>0$. Existence of four linearly independent solutions with the exponential tails in (4.5) and (4.6) follows by the Coddington-Levinson's Theorem for ODEs [10] under the condition that $V(x) \rightarrow 0$ and $\phi_{\epsilon}^{2}(x) \rightarrow q_{0}$ exponentially fast as $|x| \rightarrow \infty$.

Definition 4.5 The determinant of the four fundamental solutions in Lemma 4.4 at any $x \in \mathbb{R}$ is called the Evans function $E(\lambda, \epsilon)$ of the spectral problem (3.19), namely

$$
E(\lambda, \epsilon)=\operatorname{det}\left|\begin{array}{cccc}
u_{+} & \tilde{u}_{+} & u_{-} & \tilde{u}_{-} \\
u_{+}^{\prime} & \tilde{u}_{+}^{\prime} & u_{-}^{\prime} & \tilde{u}_{-}^{\prime} \\
w_{+} & \tilde{w}_{+} & w_{-} & \tilde{w}_{-} \\
w_{+}^{\prime} & \tilde{w}_{+}^{\prime} & w_{-}^{\prime} & \tilde{w}_{-}^{\prime}
\end{array}\right| .
$$

Remark 4.6 Because the Wronskian determinant of any four particular solutions of the ODE (3.19) is independent of $x$, the values of $E(\lambda, \epsilon)$ are independent of $x$.

Lemma 4.7 Let $\phi_{\epsilon}(x)$ be the kink mode of Definition [2.8, $\lambda=\frac{1}{2} \kappa_{-} \kappa_{+}$and $\kappa_{+}=\sqrt{4 c^{2}-\kappa_{-}^{2}}$. The four fundamental solutions and the Evans function $E(\lambda, \epsilon)$ of the spectral problem (3.19) are analytically continued in variable $\kappa_{-}$near $\kappa_{-}=0$ for sufficiently small $\epsilon$.

Proof. Let us unfold the branch point $\lambda=0$ with the transformation

$$
\lambda=\frac{\kappa_{+} \kappa_{-}}{2}, \quad c^{2}=\frac{\kappa_{+}^{2}+\kappa_{-}^{2}}{4} .
$$

The spectral problem (3.19) is rewritten explicitly as follows:

$$
\left[-\partial_{x}^{2}+\kappa_{+}^{2}+\kappa_{-}^{2}+2 V_{+}(x)\right] u=-\kappa_{+} \kappa_{-} w, \quad\left[-\partial_{x}^{2}+2 V_{-}(x)\right] w=\kappa_{+} \kappa_{-} u,
$$

where

$$
V_{+}(x)=f\left(\phi_{\epsilon}^{2}\right)-f\left(q_{0}\right)+2 \phi_{\epsilon}^{2} f^{\prime}\left(\phi_{\epsilon}^{2}\right)-2 q_{0} f^{\prime}\left(q_{0}\right)+\epsilon V(x), \quad V_{-}(x)=f\left(\phi_{\epsilon}^{2}\right)-f\left(q_{0}\right)+\epsilon V(x) .
$$

Since the ODE system (4.9) depends analytically on $\left(\kappa_{+}, \kappa_{-}\right) \in \mathbb{C}^{2}$ and the boundary conditions (4.5)(4.6) are also analytic in variables $\left(\kappa_{+}, \kappa_{-}\right)$, the four fundamental solutions are analytic on $\left(\kappa_{+}, \kappa_{-}\right) \in$ 
$\mathbb{C}^{2}$ and so is the Evans function $E(\lambda, \epsilon)$ as a determinant of analytic functions at any fixed $x \in \mathbb{R}$. The unfolding transformation (4.8) implies that the parameter $c \in \mathbb{C}$ is arbitrary. Since $c \in \mathbb{R}_{+}$is fixed, this leads to the constraint $\kappa_{+}=\sqrt{4 c^{2}-\kappa_{-}^{2}}$, which is locally analytic near $\kappa_{-}=0$. The analytic functions on $\left(\kappa_{+}, \kappa_{-}\right) \in \mathbb{C}^{2}$ with the locally analytic constraint $\kappa_{+}=\sqrt{4 c^{2}-\kappa_{-}^{2}}$ are locally analytic functions in a neighborhood of $\kappa_{-}=0$ for any sufficiently small $\epsilon$.

Remark 4.8 The Evans function was constructed in [19] for the cubic NLS equation with a perturbation. It was discussed in [19] under a general set of assumptions that the function $E(\lambda)$ is analytic in a small domain near $\lambda=0$ with $\operatorname{Re} \lambda>0$, its zeros coincide with eigenvalues $\lambda$ with the account of their algebraic multiplicities, and it is analytically continued in the variable $\kappa_{-}$near the point $\lambda=0$ $\left(\kappa_{-}=0\right)$. Our analysis of Lemma 4.7] is different. It is based on the unfolding transformation (4.8) similarly to the recent work [12].

Example 4.9 Continuing Example 2.15, we compute the Evans function $E(\lambda)$ explicitly for the cubic NLS with $f(s)=s$ and $q_{0}=1$. There exist explicit solutions of the spectral problem (3.1) for the cubic NLS (see, e.g. 25]). By using these solutions, we obtain the explicit representation of the eigenvectors $(u, w)$ satisfying the boundary conditions (4.5) and (4.6):

$$
u_{ \pm}=-\frac{2}{2+\kappa_{ \pm}} e^{\kappa_{ \pm} x}\left(\operatorname{sech}^{2} x+\kappa_{ \pm} \tanh x-\frac{1}{2} \kappa_{ \pm}^{2}\right), \quad w_{ \pm}=-\frac{\kappa_{\mp}}{2+\kappa_{ \pm}} e^{\kappa_{ \pm} x}\left(\kappa_{ \pm}-2 \tanh x\right)
$$

and

$$
\tilde{u}_{ \pm}=-\frac{2}{2+\kappa_{ \pm}} e^{-\kappa_{ \pm} x}\left(\operatorname{sech}^{2} x-\kappa_{ \pm} \tanh x-\frac{1}{2} \kappa_{ \pm}^{2}\right), \quad \tilde{w}_{ \pm}=-\frac{\kappa_{\mp}}{2+\kappa_{ \pm}} e^{-\kappa_{ \pm} x}\left(\kappa_{ \pm}+2 \tanh x\right) .
$$

The Evans function $E(\lambda)$ is computed explicitly as the determinant of the four fundamental solutions in the form

$$
E(\lambda)=\frac{4 \kappa_{+}^{3} \kappa_{-}^{3}\left(\kappa_{+}^{2}-\kappa_{-}^{2}\right)^{2}}{\left(\kappa_{+}+2\right)^{2}\left(\kappa_{-}+2\right)^{2}},
$$

such that $E(\lambda)=8 \lambda^{3}\left(1-\lambda+\mathrm{O}\left(\lambda^{2}\right)\right)$ as $\lambda \rightarrow 0$ with $\operatorname{Re} \lambda>0$. The validity of all explicit formulas has been confirmed by means of the Wolfram's Mathematica.

Theorem 4.10 Let $f(q)$ be $C^{\infty}\left(\mathbb{R}_{+}\right)$and $V(x)$ be $C^{2}(\mathbb{R})$ satisfying (1.3). Let $\left.P_{r}^{\prime}\right|_{v \downarrow 0} \neq 0$ and $M^{\prime \prime}\left(s_{0}\right)=$ 0 in Theorems 2.12 and 3.11. Let $\gamma=\Gamma(\epsilon)$ be an eigenvalue of the generalized eigenvalue problem (3.22) for sufficiently small $\epsilon$, such that $w \in L^{2}(\mathbb{R}), \operatorname{Im} \Gamma \leq 0$, and $\lim _{\epsilon \rightarrow 0} \Gamma(\epsilon)=0$. Then, the spectral problem (3.19) for sufficiently small $\epsilon$ admits two eigenvalues $\lambda= \pm \Lambda(\epsilon)= \pm \sqrt{-\Gamma(\epsilon)}$ with $(u, w) \in L^{2}\left(\mathbb{R}, \mathbb{C}^{2}\right)$, $\operatorname{Re} \Lambda \operatorname{Im} \Lambda \geq 0$, and $\lim _{\epsilon \rightarrow 0} \Lambda(\epsilon)=0$, such that

(i) $\Lambda(\epsilon)$ is infinitely smooth with respect to $\epsilon^{1 / 2}$.

(ii) $(u, w)$ is infinitely smooth with respect to $\epsilon^{1 / 2}$ and

$$
\lim _{\epsilon \rightarrow 0} u(x)=\phi_{0}^{\prime}(x), \quad \lim _{\epsilon \rightarrow 0} w(x)=0,
$$

up to an arbitrary multiplicative factor. 
(iii) $(u, w)$ admits an asymptotic expansion as $\lambda \rightarrow 0, \operatorname{Re} \lambda>0$ for large $\pm x \gg 1$ :

$$
\left(\begin{array}{c}
u \\
w
\end{array}\right) \rightarrow a_{ \pm}(1+\mathrm{O}(\lambda))\left(\begin{array}{c}
\lambda / c+\mathrm{O}\left(\lambda^{3}\right) \\
-2 c+\mathrm{O}\left(\lambda^{2}\right)
\end{array}\right)\left(1 \mp \frac{\lambda x}{c}+\mathrm{O}(\lambda x)^{2}\right)
$$

where $a_{ \pm}$are some constants and $\lambda=\Lambda(\epsilon)$.

Proof. Under the conditions of the theorem, there must exist a zero of the Evans function $E(\lambda, \epsilon)$ at $\lambda=\Lambda(\epsilon)$ for sufficiently small $\epsilon$, such that $\operatorname{Re} \Lambda \operatorname{Im} \Lambda \geq 0$ and $\lim _{\epsilon \rightarrow 0} \Lambda(\epsilon)=0$.

(i) By Lemma 4.7 the Evans function $E(\lambda, \epsilon)$ is analytically continued in $\kappa_{-}=\lambda / c+\mathrm{O}\left(\lambda^{3}\right)$ near $\lambda=0$. It is also infinitely smooth in $\epsilon$ near $\epsilon=0$. (Indeed, the potential terms $V_{ \pm}(x)$ in the representation (4.9) are infinitely smooth in $\epsilon$ and exponentially decaying as $|x| \rightarrow \infty$.) In addition, the Evans function $E(\lambda, \epsilon)$ has the following properties:

$$
E(\lambda, 0)=\alpha \lambda^{3}+\mathrm{O}\left(\lambda^{4}\right), \quad E(0, \epsilon)=0
$$

where $\alpha$ is a numerical constant. According to Lemma 4.1 the triple root of $E(\lambda, 0)$ corresponds to the generalized kernel (i) due to translational invariance and the bounded solutions (ii) due to the gauge invariance. The former subspace results in the double root $\lambda=0$ of $E(\lambda, 0)$ (as a proper eigenvalue), while the latter subspace results in a single root $\kappa_{-}=0(\lambda=0)$ of $E(\lambda, 0)$ (as a proper resonance) [20]. By Lemma 4.1, $\alpha \neq 0$ is equivalent to the condition $\left.P_{r}^{\prime}\right|_{v \downarrow 0} \neq 0$. The constraint $E(0, \epsilon)=0$ follows from existence of $\phi_{\epsilon} \in L^{\infty}(\mathbb{R})$ such that $\mathcal{L}_{-} \phi_{\epsilon}=0$. As a result, the Evans function is expanded near $\lambda=0$ and $\epsilon=0$ as follows:

$$
E(\lambda, \epsilon)=\lambda\left(\alpha \lambda^{2}+\beta \epsilon+\mathrm{O}\left(\lambda^{3}, \lambda \epsilon, \epsilon^{2}\right)\right),
$$

where $\beta$ is another numerical constant. Since the gauge invariance is preserved while the translational invariance of dark solitons is destroyed by Theorem 3.15 $\beta \neq 0$ is equivalent to the condition that $M^{\prime \prime}\left(s_{0}\right) \neq 0$. It follows from expansion (4.15) and the smoothness of $E(\lambda, \epsilon)$ that the root $\Lambda(\epsilon)$ is infinitely smooth in $\epsilon^{1 / 2}$.

(ii) By the construction of the Evans function $E(\lambda, \epsilon)$, the eigenvector $(u, w)$ is spanned by the four fundamental solutions in Lemma 4.4. By Lemma 4.7 these solutions are analytic in $\kappa_{-}$near $\kappa_{-}=0$. By Remark 4.3 $\kappa_{-}=\lambda / c+\mathrm{O}\left(\lambda^{3}\right)$ near $\lambda=0$ and by (i) of Theorem 4.10, the root $\lambda=\Lambda(\epsilon)$ of $E(\lambda, \epsilon)=0$ is infinitely smooth in $\epsilon^{1 / 2}$. Therefore, the eigenvector $(u, w)$ is also infinitely smooth in $\epsilon^{1 / 2}$. By Lemma 4.1, the kernel of operators $\mathcal{L}_{+}$and $\mathcal{L}_{-}$is one-dimensional in $L^{2}(\mathbb{R})$, such that the limiting relation (4.13) follows by smoothness (eigenvectors are defined up to an arbitrary multiplicative factor).

(iii) It follows from the decay (4.5) and (4.6) for the four fundamental solutions that there exist constants $A_{ \pm}(\lambda)$ and $B_{ \pm}(\lambda)$, such that

$$
\left(\begin{array}{c}
u \\
w
\end{array}\right) \rightarrow A_{ \pm}\left(\begin{array}{c}
\kappa_{-} \\
-\kappa_{+}
\end{array}\right) e^{\mp \kappa_{-} x}+B_{ \pm}\left(\begin{array}{c}
\kappa_{+} \\
-\kappa_{-}
\end{array}\right) e^{\mp \kappa_{+} x} \quad \text { as } \quad x \rightarrow \pm \infty
$$

where $\kappa_{ \pm}$are defined by the characteristic equation (4.3) with $\lambda=\Lambda(\epsilon)$. By expansion (4.4), the expansion of slowly decaying solutions $e^{\mp \kappa_{-} x}$ gives (4.14), while expansion of fast decaying solutions $e^{\mp \kappa+x}$ is not written. 
Theorem 4.11 Let $f(q)$ be $C^{2}\left(\mathbb{R}_{+}\right)$and $V(x)$ satisfy (1.3). Let $\left.P_{r}^{\prime}\right|_{v \downarrow 0} \neq 0$ and $\left.S^{\prime}\right|_{v \downarrow 0} \neq 0$ for the black soliton $\phi_{0}(x)$ of Definition [2.5. Let $\lambda=\Lambda(\epsilon)$ be a small eigenvalues of the spectral problem (3.19) for sufficiently small $\epsilon$ with $\operatorname{Re} \Lambda>0$. Then, the value of $\Lambda(\epsilon)$ is given by the root of the characteristic equation:

$$
\operatorname{Re} \Lambda>0: \quad\left(\left.P_{r}^{\prime}\right|_{v \downarrow 0}\right) \Lambda^{2}-\epsilon \frac{q_{0}\left(\left.S^{\prime}\right|_{v \downarrow 0}\right)^{2} M^{\prime \prime}\left(s_{0}\right)}{2 c\left(\left.P_{r}^{\prime}\right|_{v \downarrow 0}\right)} \Lambda+\epsilon M^{\prime \prime}\left(s_{0}\right)=\mathrm{O}\left(\epsilon^{2}\right) .
$$

Proof. By Remark 3.16 the spectral problem (3.19) for sufficiently small $\epsilon$ can be written in the form:

$$
\left[L_{+}+\epsilon M_{+}+\mathrm{O}\left(\epsilon^{2}\right)\right] u=-\lambda w, \quad\left[L_{-}+\epsilon M_{-}+\mathrm{O}\left(\epsilon^{2}\right)\right] w=\lambda u .
$$

By Theorem 4.10, the eigenvector $(u, w)$ and the eigenvalue $\lambda=\Lambda(\epsilon)$ can be expanded in powers of $\epsilon^{1 / 2}$ :

$$
u=\phi_{0}^{\prime}(x)+\epsilon^{1 / 2} u_{1}+\epsilon u_{2}+\epsilon^{3 / 2} u_{3}+\mathrm{O}\left(\epsilon^{2}\right), \quad w=\epsilon^{1 / 2} w_{1}+\epsilon w_{2}+\epsilon^{3 / 2} w_{3}+\mathrm{O}\left(\epsilon^{2}\right),
$$

and $\lambda=\epsilon^{1 / 2} \lambda_{1}+\epsilon \lambda_{2}+\epsilon^{3 / 2} \lambda_{3}+\mathrm{O}\left(\epsilon^{2}\right)$. The first-order corrections terms satisfy the system

$$
L_{+} u_{1}=0, \quad L_{-} w_{1}=\lambda_{1} \phi_{0}^{\prime} .
$$

By the expansion (4.14), we are looking for a solution with $u_{1} \in L^{2}(\mathbb{R})$ and $w_{1} \in L^{\infty}(\mathbb{R})$. By Lemma 4.1. the explicit solution is

$$
u_{1}=c_{1} \phi_{0}^{\prime}(x), \quad w_{1}=a_{1} \phi_{0}(x)-\left.\lambda_{1} \operatorname{Im} \partial_{v} U(x)\right|_{v \downarrow 0},
$$

where $\left(a_{1}, c_{1}\right)$ are parameters. Without loss of generality, we can set $c_{1}=0$. The second-order corrections terms satisfy the system

$$
L_{+} u_{2}=-\lambda_{1} w_{1}-M_{+} \phi_{0}^{\prime}, \quad L_{-} w_{2}=\lambda_{2} \phi_{0}^{\prime}
$$

By the Fredholm alternative for $L_{+}$, we obtain the constraint

$$
-\lambda_{1}\left(\phi_{0}^{\prime}, w_{1}\right)-\left(\phi_{0}^{\prime}, M_{+} \phi_{0}^{\prime}\right)=0 .
$$

By using (3.14) and (3.25), the constraint is equivalent to the characteristic equation

$$
\frac{1}{2}\left(\left.P_{r}^{\prime}\right|_{v \downarrow 0}\right) \lambda_{1}^{2}+\frac{1}{2} M^{\prime \prime}\left(s_{0}\right)=0 .
$$

Since $w_{1} \in L^{\infty}(\mathbb{R})$ and the Fredholm constraint is satisfied, there exists a solution $u_{2} \in L^{\infty}(\mathbb{R})$ of the first inhomogeneous equation (4.19). There exists also a solution $w_{2} \in L^{\infty}(\mathbb{R})$ of the second inhomogeneous equation (4.19) similarly to the solution $w_{1} \in L^{\infty}(\mathbb{R})$. By the expansion (4.14), we shall add a homogeneous linearly growing solution (iii) of Lemma 4.1 which is not in $L^{\infty}(\mathbb{R})$, such that $w_{2} \notin L^{\infty}(\mathbb{R})$. As a result, the second-order corrections terms are written in the form

$$
\begin{aligned}
u_{2} & =c_{2} \phi_{0}^{\prime}(x)+\tilde{u}_{2}(x), \\
w_{2} & =a_{2} \phi_{0}(x)-\left.\lambda_{2} \operatorname{Im} \partial_{v} U(x)\right|_{v \downarrow 0}+b_{2}\left(x \phi_{0}(x)-\left.\operatorname{Im} \partial_{v} U(x)\right|_{v \downarrow 0}\right),
\end{aligned}
$$

where $\left(a_{2}, b_{2}, c_{2}\right)$ are parameters $\left(c_{2}=0\right.$ without loss of generality) and $\tilde{u}_{2} \in L^{\infty}(\mathbb{R})$ is a solution of the inhomogeneous equation

$$
L_{+} \tilde{u}_{2}=-\lambda_{1} a_{1} \phi_{0}-\left.\frac{M^{\prime \prime}\left(s_{0}\right)}{\left.P_{r}^{\prime}\right|_{v \downarrow 0}} \operatorname{Im} \partial_{v} U(x)\right|_{v \downarrow 0}-M_{+} \phi_{0}^{\prime} .
$$


The third-order corrections terms satisfy the system

$$
L_{+} u_{3}=-\lambda_{2} w_{1}-\lambda_{1} w_{2}, \quad L_{-} w_{3}=\lambda_{3} \phi_{0}^{\prime}+\lambda_{1} u_{2}-M_{-} w_{1} .
$$

By the Fredholm alternative for $L_{+}$, we obtain the constraint

$$
-\lambda_{2}\left(\phi_{0}^{\prime}, w_{1}\right)-\lambda_{1}\left(\phi_{0}^{\prime}, w_{2}\right)=0
$$

which is equivalent by virtue of (4.2) to the characteristic equation

$$
\left(\left.P_{r}^{\prime}\right|_{v \downarrow 0}\right) \lambda_{1} \lambda_{2}+\frac{q_{0}\left(\left.S^{\prime}\right|_{v \downarrow 0}\right)}{2} \lambda_{1} b_{2}=0 .
$$

Combining two corrections $\lambda_{1}$ and $\lambda_{2}$ in $\Lambda(\epsilon)=\epsilon^{1 / 2} \lambda_{1}+\epsilon \lambda_{2}+\mathrm{O}\left(\epsilon^{3 / 2}\right)$, we rewrite the characteristic equations (4.20) and (4.22) in the form

$$
\left(\left.P_{r}^{\prime}\right|_{v \downarrow 0}\right) \Lambda^{2}+\epsilon q_{0}\left(\left.S^{\prime}\right|_{v \downarrow 0}\right) b_{2} \Lambda+\epsilon M^{\prime \prime}\left(s_{0}\right)=\mathrm{O}\left(\epsilon^{2}\right) .
$$

In order to find $b_{2}$ for $\operatorname{Re} \Lambda>0$, we need to consider $w(x)=\epsilon^{1 / 2} w_{1}+\epsilon w_{2}+\mathrm{O}\left(\epsilon^{3 / 2}\right)$ for large $\pm x \gg 1$ :

$$
w(x) \rightarrow \pm \sqrt{q_{0}}\left(\epsilon^{1 / 2}\left(a_{1}-\left.\lambda_{1} \partial_{v} \Theta^{ \pm}\right|_{v \downarrow 0}\right)+\epsilon\left(a_{2}-\left.\lambda_{2} \partial_{v} \Theta^{ \pm}\right|_{v \downarrow 0}+b_{2}\left(x-\left.\partial_{v} \Theta^{ \pm}\right|_{v \downarrow 0}\right)\right)+\mathrm{O}\left(\epsilon^{3 / 2}\right)\right)
$$

where $\Theta^{ \pm}=\lim _{x \rightarrow \pm \infty} \Theta(x)$ and $\lim _{x \rightarrow \pm \infty} \phi_{0}(x)= \pm \sqrt{q_{0}}$ have been used. Matching the asymptotic expansions (4.14) and (4.24), we find a linear system on parameters $\left(a_{1}, b_{2}\right)$ :

$$
c b_{2}=\mp \lambda_{1}\left(a_{1}-\left.\lambda_{1} \partial_{v} \Theta^{ \pm}\right|_{v \downarrow 0}\right) .
$$

The linear system has the explicit solution

$$
2 c b_{2}=\left.\lambda_{1}^{2} \partial_{v}\left(\Theta^{+}-\Theta^{-}\right)\right|_{v \downarrow 0}, \quad 2 \lambda_{1} a_{1}=\left.\lambda_{1}^{2} \partial_{v}\left(\Theta^{+}+\Theta^{-}\right)\right|_{v \downarrow 0},
$$

such that

$$
b_{2}=\frac{\left(\left.S^{\prime}\right|_{v \downarrow 0}\right)}{2 c} \lambda_{1}^{2}=-\frac{\left(\left.S^{\prime}\right|_{v \downarrow 0}\right) M^{\prime \prime}\left(s_{0}\right)}{2 c\left(\left.P_{r}^{\prime}\right|_{v \downarrow 0}\right)},
$$

where $S=\Theta^{+}-\Theta^{-}$. As a result, the characteristic equation (4.23) reduces to the form (4.17).

Corollary 4.12 Let $\left.P_{r}^{\prime}\right|_{v \downarrow 0} \neq 0$ and $\left.S^{\prime}\right|_{v \downarrow 0} \neq 0$ for the kink mode of Theorems [2.12, 3.14, 3.15 and 4.11 for sufficiently small $\epsilon$. Then,

- If $\left.P_{r}^{\prime}\right|_{v \downarrow 0}>0$, the kink mode with $M^{\prime \prime}\left(s_{0}\right)>0$ has precisely one quartet of small complex eigenvalues $\left(N_{r}=N_{i}^{-}=0, N_{c}=1\right)$, while the kink mode with $M^{\prime \prime}\left(s_{0}\right)<0$ has precisely one pair of small real eigenvalues $\left(N_{r}=1, N_{i}^{-}=N_{c}=0\right)$ in the spectral problem (3.19).

- If $\left.P_{r}^{\prime}\right|_{v \downarrow 0}<0$, the kink mode with $M^{\prime \prime}\left(s_{0}\right)>0$ has precisely one pair of small real eigenvalues and one pair of finite real eigenvalues $\left(N_{r}=2, N_{i}^{-}=N_{c}=0\right)$, while the kink mode with $M^{\prime \prime}\left(s_{0}\right)<0$ has precisely one pair of finite real eigenvalues and no small eigenvalues $\left(N_{r}=1, N_{i}^{-}=N_{c}=0\right)$ in the spectral problem (3.19). 
Remark 4.13 Comparison of the characteristic equation (4.17) and the linearized version of the Newton's particle law (1.7) shows that

$$
\mu_{0}=\left.P_{r}^{\prime}\right|_{v \downarrow 0}, \quad \lambda_{0}=\frac{q_{0}\left(\left.S^{\prime}\right|_{v \downarrow 0}\right)^{2}}{2 c\left(\left.P_{r}^{\prime}\right|_{v \downarrow 0}\right)} .
$$

Both constants are positive if $\left.P_{r}^{\prime}\right|_{v \downarrow 0}>0$, i.e. if the kink is stable in the spectral problem (3.1).

Remark 4.14 Characteristic equation (4.17) can be derived from the power expansion of the Evans function $E(\lambda, \epsilon)$ of Definition 4.5 .

$$
E(\lambda, \epsilon)=\lambda\left(\alpha \lambda^{2}+\tilde{\alpha} \lambda^{3}+\beta \epsilon+\tilde{\beta} \lambda \epsilon+\mathrm{O}\left(\lambda^{4}, \lambda^{2} \epsilon, \epsilon^{2}\right)\right)
$$

where $(\alpha, \beta)$ are constants from the expansion (4.15) and $(\tilde{\alpha}, \tilde{\beta})$ are new constants. Computations of these constants from derivatives of $E(\lambda, \epsilon)$ are technically involved (see [19, 44]). These computations are replaced in Theorem 4.11 with direct expansions of eigenvectors and eigenvalues of the spectral problem (3.19) in powers of $\epsilon^{1 / 2}$.

Example 4.15 Continuing Example 3.7 we consider the cubic NLS with $f(s)=s$ and $q_{0}=1$, where $\left.P_{r}^{\prime}\right|_{v \downarrow 0}=4$ and $\left.S^{\prime}\right|_{v \downarrow 0}=2$. As a result, the characteristic equation (4.17) is written explicitly by

$$
\operatorname{Re} \Lambda>0: \quad \Lambda^{2}+\frac{\epsilon}{4} M^{\prime \prime}\left(s_{0}\right)\left(1-\frac{\Lambda}{2}\right)=\mathrm{O}\left(\epsilon^{2}\right)
$$

This equation has only one real-valued root $\Lambda(\epsilon)>0$ for $M^{\prime \prime}\left(s_{0}\right)<0$ and two complex-conjugate roots with $\operatorname{Re} \Lambda(\epsilon)>0$ for $M^{\prime \prime}\left(s_{0}\right)>0$ provided that $\epsilon>0$ is sufficiently small. The validity of the expansion (4.26) will be tested in Section [5.

Remark 4.16 If the characteristic equation (4.26) is formally applied to the cubic GP equation (1.2) with $f(s)=s, q_{0}=1$ and $V(x)=x^{2}$, we obtain $M^{\prime \prime}(s)=2 \int_{\mathbb{R}} \operatorname{sech}^{2}(x) d x=4$, such that the characteristic equation (4.26) is

$$
\Lambda^{2}-\frac{\epsilon}{2} \Lambda+\epsilon=\mathrm{O}\left(\epsilon^{2}\right)
$$

This characteristic equation was derived in 34] with a formal method for slow dynamics of dark solitons in parabolic potentials subject to radiative boundary conditions. The validity of radiative boundary conditions for parabolic potentials $V(x)$ can not be verified by the present analysis.

Remark 4.17 The characteristic equation (4.17) can be rewritten in the form

$$
\left(\left.P_{r}^{\prime}\right|_{v \downarrow 0}\right) \Lambda^{2}+\frac{q_{0}\left(\left.S^{\prime}\right|_{v \downarrow 0}\right)^{2}}{2 c} \Lambda^{3}=-\epsilon M^{\prime \prime}\left(s_{0}\right)+\mathrm{O}\left(\epsilon^{2}\right)
$$

The left-hand-side of this equation was derived in Eqs. (19)-(20) of [35] and Eqs. (2.37)-(2.38) of [33] in a more general context of dark solitons with $v \in(-c, c)$. The method of 35] was based on asymptotic theory for slow dynamics of dark solitons, while the method of [33] was based on slow decay conditions for eigenfunctions of the linearized problem. Here we have replaced these formal methods with rigorous proof in the framework of Theorems 4.10 and 4.11 


\section{$5 \quad$ Numerical approximations of eigenvalues}

We test here the predictions of the characteristic equation (4.26) for the cubic GP equation (1.2) with $f(s)=s$ and the two potentials $V_{1}(x)$ and $V_{2}(x)$ in (1.4). In particular, we focus on examining the dependencies of small unstable eigenvalues of the spectral problem (3.19) versus parameters $\epsilon$ and $\kappa$. The numerical approximations of the kink mode $\phi_{\epsilon}(x)$ are obtained by means of fixed point iterations of the ODE (2.12). The iterations are applied to a finite-difference discretization of the computational domain $x \in[-L, L]$ (typically $L=10$ ) on a grid of $N$ nodes (typically $N=1600$ ) with a spacing $\Delta x$ (typically $\Delta x=0.2$ ). Subsequently, the spectral problem (3.19) is discretized in a matrix eigenvalue problem that, in turn, is solved through standard numerical linear algebra routines.

In the case of the potential $V_{1}(x)$, as is considered in Examples 2.15 and 3.19 the positive-definite sign of $L^{\prime \prime}(0)$ (and hence $M^{\prime \prime}(0)$ ) leads to a sole kink mode bifurcating from $s_{0}=0$ (and staying at $s=0$ by Remark 2.10). The kink mode is located at the minimum of the effective potential $M(s)$ and is unstable due to a complex quartet of eigenvalues according to the characteristic equation (4.26).

The numerical results on Figures 2 and 3 fully confirm the above picture. Fig. 2 shows only one solution $\phi_{\epsilon}(x)$ of the ODE (2.12) with the potential $V_{1}(x)$ and a unique quartet of complex eigenvalues $\lambda=\lambda_{r}+i \lambda_{i}$ in the spectral problem (3.19). The left panel of Fig. 3 shows the real part of this quartet as a function of $\kappa$ for a given $\epsilon=0.2$, while the right panel shows the relevant real part as a function of $\epsilon$ for a given $\kappa=1$. The predictions of the characteristic equation (4.26) are shown by dashed-dotted lines, while the numerically obtained eigenvalues are shown by thick lines.

The non-monotonic behavior of the real part of complex eigenvalues is produced by the truncation of the computational domain $x \in[-L, L]$ and subsequent discretization on a finite grid. This numerical phenomenon is explained in [17] (see their Figure 2) as follows. The continuous spectrum of the spectral problem (3.19) becomes a finite spectral band along the imaginary axis near $\lambda=0$ due to the truncation and the band is represented by isolated eigenvalues due to the discretization. The quartet of eigenvalues bifurcates from the point $\lambda=0$ in the direction of the imaginary axis with small real part for small $\epsilon$ and interferes with eigenvalues from the discretized continuous spectrum. This interference leads to the non-monotonic behavior of the real part of the relevant eigenvalues on Fig. 3. We note that this effect is not present for real eigenvalues bifurcating from the point $\lambda=0$.

In the case of the potential $V_{2}(x)$, we have a more interesting phenomenology. While the potential always has two maxima at $x= \pm 2 / \kappa$, the effective potential $M(s)$ possesses two maxima at $s_{0}= \pm s_{*}$, $s_{*} \neq 0$ and a minimum at $s_{0}=0$ for $\kappa<\kappa_{0} \approx 3.21$ and only one maximum at $s_{0}=0$ for $\kappa>\kappa_{0}$. Branches of solutions with $M^{\prime \prime}\left(s_{0}\right)<0$ are unstable due to a pair of real eigenvalues, while the branch of solutions with $M^{\prime \prime}\left(s_{0}\right)>0$ is unstable due to a quartet of complex eigenvalues, according to the characteristic equation (4.26). The transition of the kink mode $s_{0}=0$ from $\kappa<\kappa_{0}\left(\right.$ when $\left.M^{\prime \prime}(0)>0\right)$ to $\kappa>\kappa_{0}\left(\right.$ when $\left.M^{\prime \prime}(0)<0\right)$ resembles the subcritical pitchfork bifurcation at $\kappa=\kappa_{0}$.

The numerical results for the potential $V_{2}(x)$ are shown on Figures 4 , 5 and 6. Branches of the solutions with $s_{0}= \pm s_{*}$ are denoted by a dashed line, the branch of the solution $s_{0}=0$ with $M^{\prime \prime}\left(s_{0}\right)>0$ is denoted by thick solid line and the same branch with $M^{\prime \prime}\left(s_{0}\right)<0$ is denoted by thick dashed line. The corresponding predictions from the extremal points of the effective potential $M(s)$ and from the characteristic equation (4.26) are shown by dash-dotted lines (thick for $s_{0}=0$ and thin for $s_{0}= \pm s_{*}$ ).

The bifurcation point is found numerically to be $\kappa_{0} \approx 3.26$ for $\epsilon=0.2$ in a very good agreement with the value $\kappa_{0}=3.21$ obtained from $M(s)$ with $M^{\prime \prime}(0)=0$. The computational error is approximately 

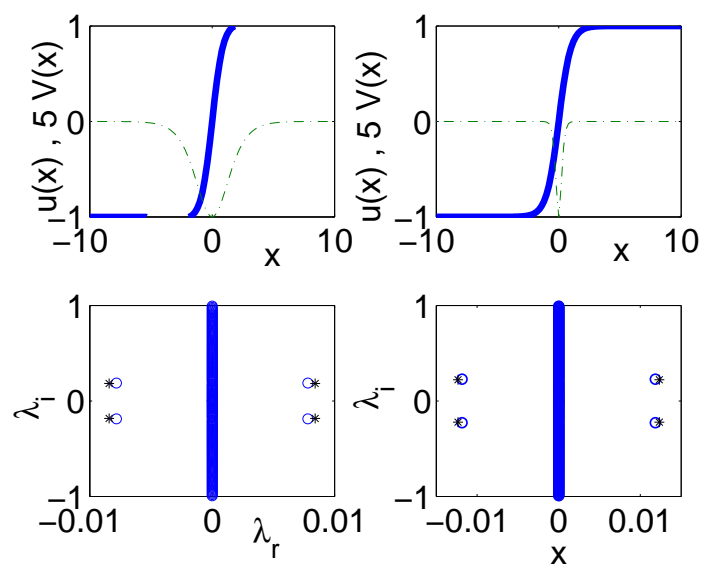

Figure 2: Bifurcation results for the potential $V_{1}(x)$. The top panels show the kink mode (solid line) and the potential $V_{1}(x)$ amplified by a factor of 5 (dash-dotted line) for $(\epsilon, \kappa)=(0.2,1.1)$ (left) and $(\epsilon, \kappa)=(0.2,6.4)$ (right). The bottom panels show the corresponding spectrum of the linearized problem (the numerical result is shown by circles, while the theoretical prediction is shown by stars).
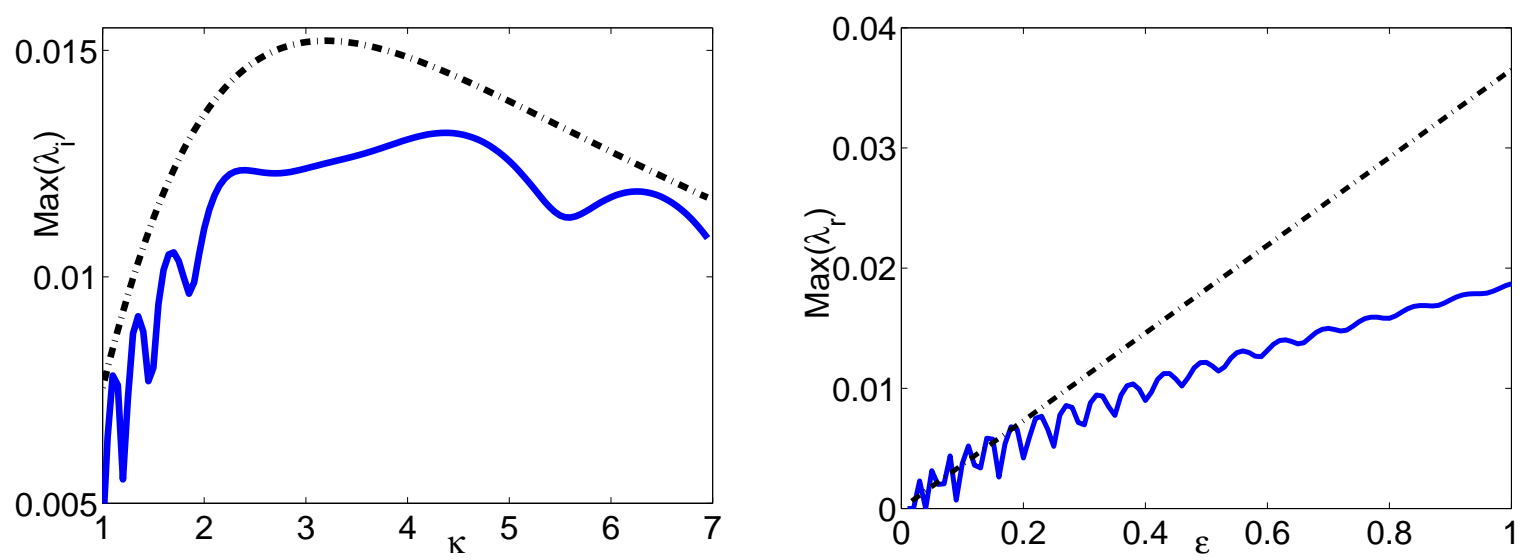

Figure 3: The left panel shows the theoretical (dash-dotted line) and numerical (solid line) dependence on $\kappa$ of the real part of the unstable complex eigenvalue for fixed $\epsilon=0.2$. The right panel shows the dependence of the same quantity on $\epsilon$ for fixed $\kappa=1$. 

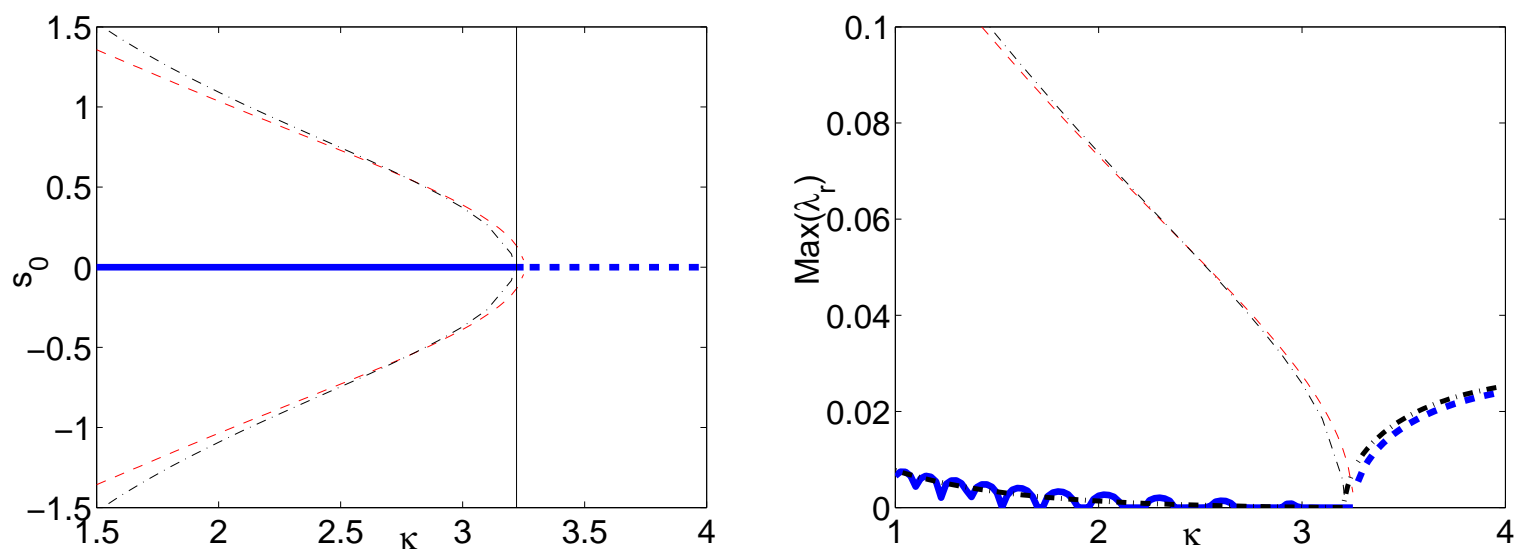

Figure 4: The subcritical pitchfork bifurcation in parameter $\kappa$ for the potential $V_{2}(x)$ for fixed $\epsilon=0.2$. The left panel shows the center of mass $s_{0}$ of the kink modes $\left(s_{0} \neq 0\right.$ by dashed line, $s_{0}=0$ by thick solid and dashed lines). The theoretical predictions of $s_{0}$ are shown by dash-dotted line. The vertical line gives the theoretical prediction for the bifurcation point $\kappa=\kappa_{0}$. The right panel shows the real part of the unstable eigenvalues for the relevant kink modes, using the same symbolism as the left panel. The theoretical predictions of eigenvalues are shown by thick and thin dash-dotted lines, respectively for the branches with $s_{0}=0$ and $s_{0} \neq 0$.

1.5\%. The values of $s_{0}$ for the kink mode $\phi_{\epsilon}(x)$ are obtained numerically from its "center of mass" defined by

$$
s_{0}=\frac{\int_{-\infty}^{\infty} x\left(1-\left|\varphi_{\epsilon}\right|^{2}\right) d x}{\int_{-\infty}^{\infty}\left(1-\left|\varphi_{\epsilon}\right|^{2}\right) d x} .
$$

The values of $s_{0}$ are plotted on the left panel of Fig. 4 for $\epsilon=0.2$ in a good agreement with the value $s_{*}$ obtained from $M(s)$ with $M^{\prime}\left(s_{*}\right)=0$.

The solution profile $\phi_{\epsilon}(x)$ and the corresponding linearization spectra for the different branches and for particular choices of $(\epsilon, \kappa)$ are shown on Fig. 5. In agreement with the characteristic equation (4.26), the kink modes with $s_{0}= \pm s_{*}$ for $\kappa<\kappa_{0}$ and with $s_{0}=0$ for $\kappa>\kappa_{0}$ has a pair of small real eigenvalues, while the kink mode with $s_{0}=0$ for $\kappa<\kappa_{0}$ has a quartet of small complex eigenvalues. The quartet of complex eigenvalues for the kink mode with $s_{0}=0$ and the pair of real eigenvalues for the kink mode with $s_{0} \neq 0$ exists for $\kappa<\kappa_{0}$, merge at the origin at $\kappa=\kappa_{0}$ and lead to a pair of real eigenvalues for the kink mode with $s_{0}=0$ and $\kappa>\kappa_{0}$. The right panel of Fig. [4 shows the real parts of unstable eigenvalues for each kink mode.

Fig. [6] shows the dependence of the relevant eigenvalues versus $\epsilon$ for a fixed $\kappa=1<\kappa_{0}$. In this case, three branches of kink modes exist and the branch with $s_{0} \neq 0$ has a pair of real eigenvalues, while the branch with $s_{0}=0$ has a quartet of complex eigenvalues. We can see that the non-monotonic behavior of the real part of unstable eigenvalues is only observed for the quartet of complex eigenvalues. We can also see from all figures of this section that the agreement between numerical and theoretical results is excellent for $\epsilon<0.3$ and deteriorate for $\epsilon>0.3$ due to the truncation error of the order of $\mathrm{O}\left(\epsilon^{2}\right)$ in the characteristic equation (4.26). 

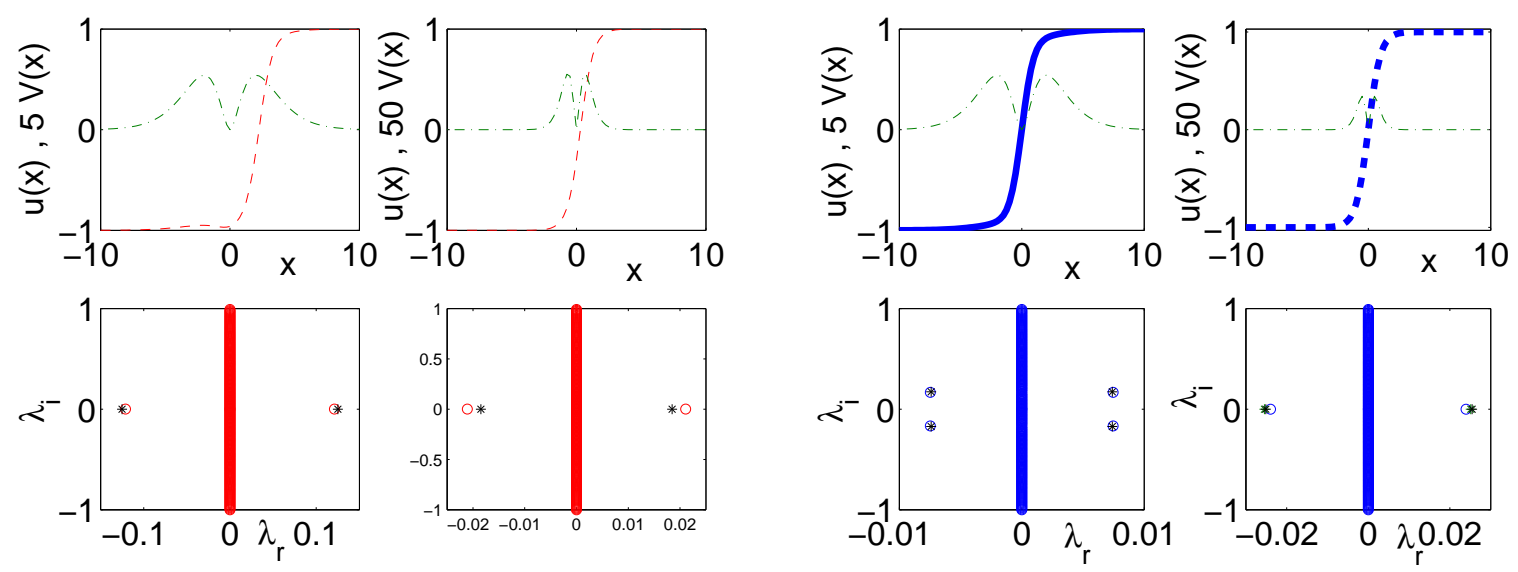

Figure 5: The left quartet of panels shows the solutions with $s_{0} \neq 0$ (dashed lines) and the potential $V_{2}(x)$ (dash-dotted line) for $(\epsilon, \kappa)=(0.2,1.0)$ and $(\epsilon, \kappa)=(0.2,3.1)$. The corresponding spectrum features a pair of real eigenvalues (numerical results are shown by circles and the theoretical predictions are shown by stars). The right quartet of panels shows the similar picture for the kink mode with $s_{0}=0$ for $(\epsilon, \kappa)=(0.2,1.025)$ and $(\epsilon, \kappa)=(0.2,3.975)$. The corresponding spectrum features either a quartet of complex eigenvalues or a pair of real eigenvalues.
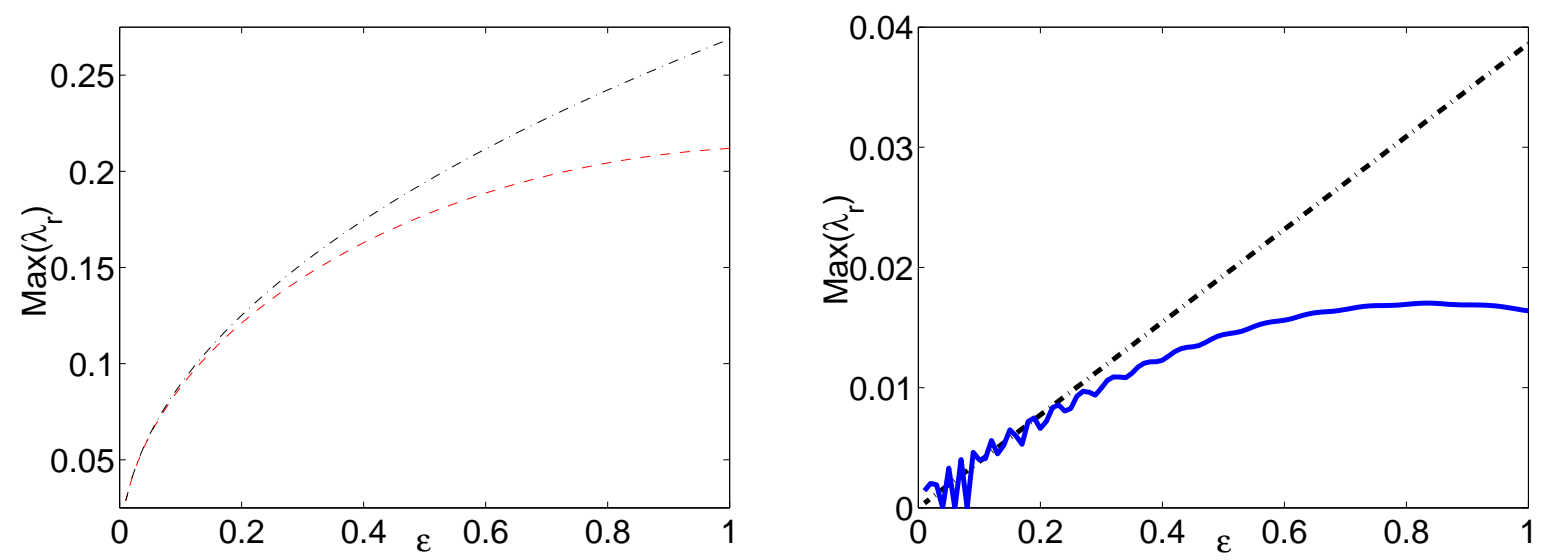

Figure 6: The real part of the unstable eigenvalues versus $\epsilon$ for $\kappa=1$ for the solution branches with $s_{0} \neq 0$ (left panel) and with $s_{0}=0$ (right panel). The numerical results are shown by dashed and thick solid lines, while the theoretical predictions are shown by dash-dotted lines. 


\section{Numerical simulations of the GP equation}

We examine here the dynamics of the unstable kink modes in the full GP equation (1.2) by using direct numerical simulations. The time-evolution problem is approximated by the fourth-order Runge-Kutta method applied to the spatial discretization of the GP equation. The output of the fixed point iteration was used as input in the time evolution integrator with the time step $\Delta t$ (typically $\Delta t=0.001$ ). The results of the time evolution are compared against the effective Newton's particle equation (1.7) for the position $s(t)$ of the center of dark soliton $\phi_{\epsilon}(x-s(t))$.

In order to test the theoretical result, we have to use the following numerical technique. The initial condition $u(x, 0)$ of the GP equation (1.2) is specified in the form of the kink mode $\phi_{\epsilon}(x)$ plus a small (typically $10^{-4}$ ) perturbation multiple of its most unstable eigenmode. The time-evolution problem is integrated for an initial period $0<t<t_{0}$, during which the dark soliton acquires a small speed due to instabilities, which quickly grows for $t>t_{0}$. At the time instance $t=t_{0}$, we approximate the values of $s\left(t_{0}\right)$ and $\dot{s}\left(t_{0}\right)$ by using the center of mass (5.1) at $t=t_{0}$ and at earlier time instances. The Newton's particle equation (1.7) is initialized at $t=t_{0}$ with given $s\left(t_{0}\right)$ and $\dot{s}\left(t_{0}\right)$ and then integrated with the fourth-order Runge-Kutta method.

Figures 7 and 8 illustrate the time evolution of an unstable dark soliton, which possesses a pair of real eigenvalues in the linearization spectrum. Fig. 7 corresponds to the potential $V_{2}(x)$ with $\kappa=4>\kappa_{0}$, when the kink mode with $s_{0}=0$ has a real eigenvalue $\lambda=0.0241$ (the theoretical prediction of the linearized Newton's particle equation is $\lambda \approx 0.0253$ ). We observe from the figure that the unstable kink mode undertakes a monotonic transition to a stable dark soliton, which escapes the double-humped potential $V_{2}(x)$ and travels with an asymptotically constant speed. The predictions of the Newton's particle equation shown by thick dash-dotted line captures the entire process accurately but slightly precedes the full time-evolution of the GP equation. The latter discrepancy can be attributed to the larger values of $\lambda$ for the unstable eigenvalues.

Fig. 8 shows a similar monotonic transition for the potential $V_{2}(x)$ with $\kappa=1$, when the kink mode with $s_{0}=s_{*} \approx 2.23$ has a pair of real unstable eigenvalues. In this case, the theoretical prediction $\lambda \approx 0.1251$ exceeds the numerically obtained value $\lambda=0.1211$ too and the prediction of the Newton's particle equation precedes its counterpart from the GP equation. It is worth to note the qualitative agreement between the two time evolutions, including the small "leg" formed in the trajectory as the dark soliton passes the unstable kink mode with $s_{0}=-s_{*} \approx-2.23$.

Figures 9 and 10 illustrate the time evolution of an unstable dark soliton, which possesses a quartet of complex eigenvalues in the linearization spectrum. Fig. 9 corresponds to the potential $V_{2}(x)$ with $\kappa=1$, when the kink mode with $s_{0}=0$ has a quartet of complex eigenvalues with the real part $\operatorname{Re} \lambda=$ 0.06606 (the theoretical prediction of the linearized Newton's particle equation is $\operatorname{Re} \lambda \approx 0.07724$ ). We observe from the figure that the unstable kink mode oscillates in a local potential well of the doublehumped potential $V_{2}(x)$ with an increasing amplitude due to unstable complex eigenvalues. When the oscillations reach a large amplitude, the dark soliton escapes the maximum of the effective potential and transforms to a steadily moving soliton. The predictions of the Newton's particle equation represent this dynamics correctly with a larger deviation from the full GP equation in comparison with the case of monotonic transitions.

Fig. 10 shows a similar oscillatory behavior of an unstable kink mode with $s_{0}=0$ for the potential $V_{1}(x)$ with $\kappa=6.4$. In this case, the theoretical prediction $\operatorname{Re} \lambda \approx 0.0123$ exceeds again the numerically obtained value $\operatorname{Re} \lambda=0.0118$ and the prediction of the Newton's particle equation precedes its 


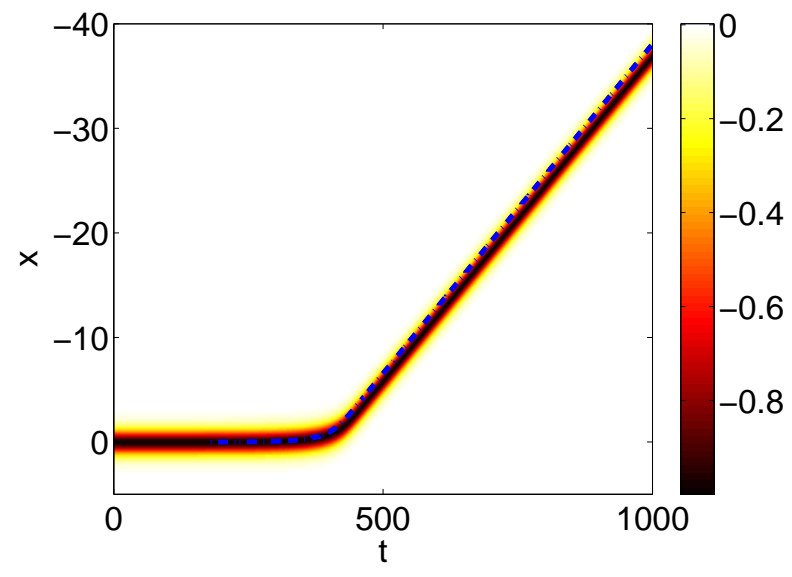

Figure 7: The unstable evolution of the kink mode with $s_{0}=0$ for the potential $V_{2}(x)$ with $\kappa=4$ and $\epsilon=0.2$. The dashed-dotted line shows the result of the Newton's particle law initialized around $t=180$.

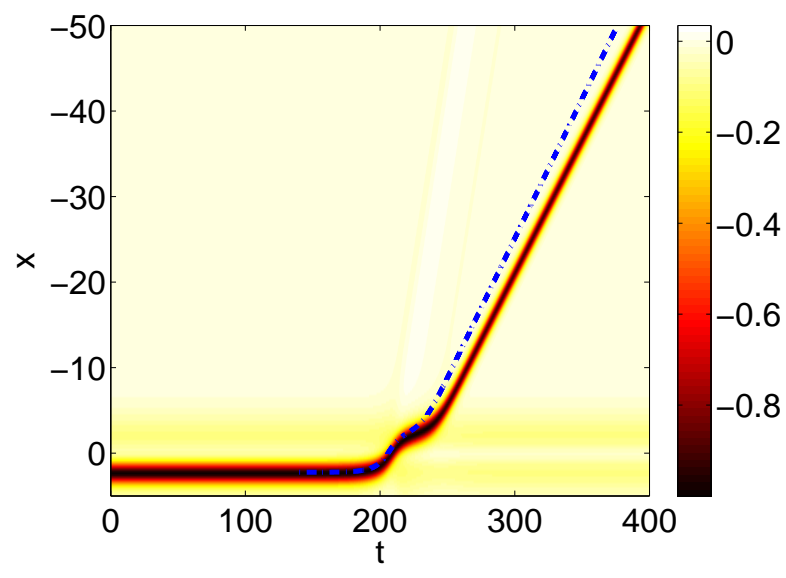

Figure 8: The unstable evolution of the kink mode with $s_{0} \approx 2.23$ for the potential $V_{2}(x)$ with $\kappa=1$ and $\epsilon=0.2$. The dashed-dotted line shows the result of the Newton's particle law initialized around $t=140$. 


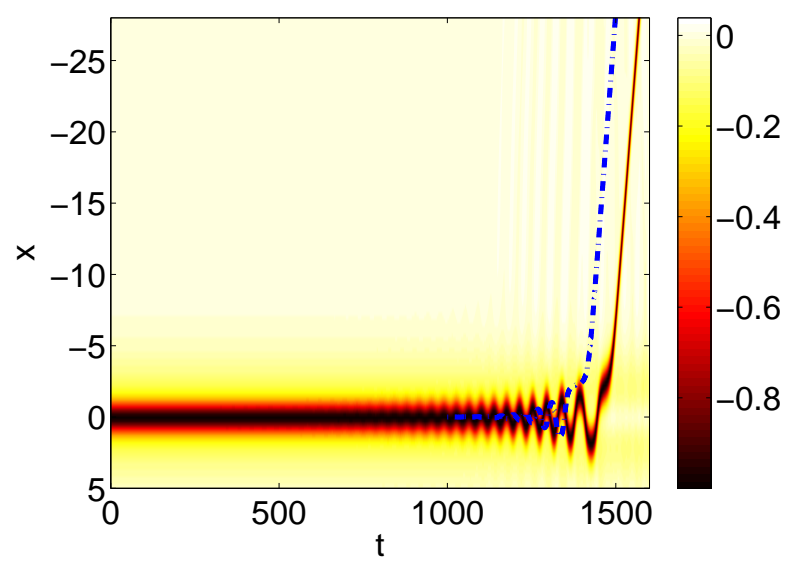

Figure 9: The unstable evolution of the kink mode with $s_{0}=0$ for the potential $V_{2}(x)$ with $\kappa=1$ and $\epsilon=0.2$. The dashed-dotted line shows the result of the Newton's particle law initialized at $t \approx 1000$.

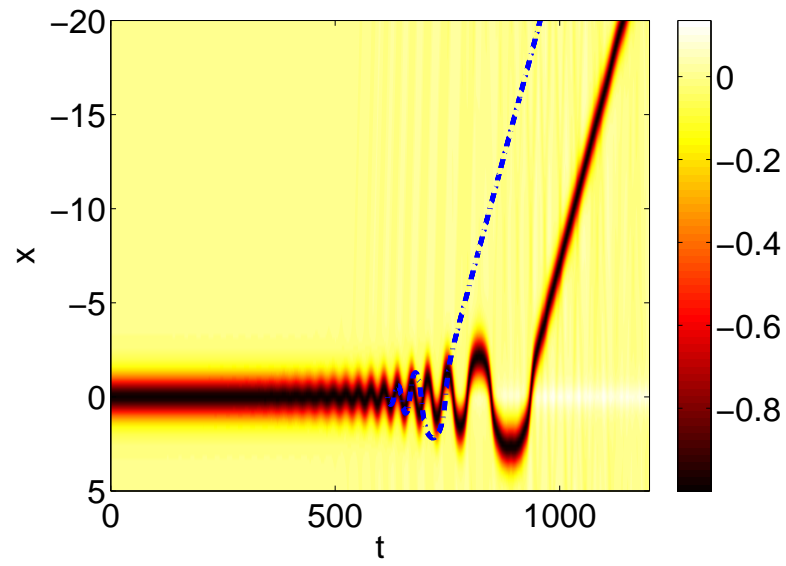

Figure 10: The unstable evolution of the kink mode with $s_{0}=0$ for the potential $V_{1}(x)$ with $\kappa=6.4$ and $\epsilon=0.2$. The dash-dotted line shows the result of the Newton's particle law initialized around $t \approx 600$. 
counterpart from the GP equation.

In the end, we mention that the rigorous derivation of the Newton's particle equation for slow dynamics of a bright soliton in an external potential has been reported recently in [6, 13, 16. Derivation of its counterpart (1.7) for slow dynamics of a dark soliton is an open problem of analysis. Our numerical results suggest that this Newton's particle equation is highly appropriate for understanding the nonlinear time-evolution of dark solitons in the GP equation with small external potentials.

\section{Conclusion}

We have systematically analyzed the persistence and stability of dark solitons in the presence of small decaying potentials. We have shown how the effective potential can be used to predict bifurcations of kink modes in a small potential and to approximate small unstable eigenvalues of the linearization spectrum. These theoretical results have been tested against the numerical bifurcation results indicating excellent qualitative and good quantitative agreement. We have also conjectured a dynamical evolution equation (the Newton's particle law) that can be used, quite successfully, to describe the motion of the kink modes and the manifestation of their instabilities.

One of the directions of interest for future studies is to expand the present results to other types of potentials which include periodic and confining potentials. While, as argued in the text, we expect many of the qualitative features to persist, periodic or growing potentials may possess additional interesting properties due to the presence of spectral bands or purely discrete spectrum in the linearization problem.

Another open direction would involve extending the present analysis to the two-dimensional setting and, in particular, to the case of vortices in the presence of external potentials. While some of the techniques applied herein (in particular, ones involving perturbative expansions) would apply to the latter case as well, others are more geared towards the one-dimensional setting (e.g. the Evans function technique). It would be especially interesting to generalize our current results to the two-dimensional GP equation.

Acknowledgement. This work was initiated during the workshop "Gross-Pitaevskii equation and related equations with non-zero boundary conditions at infinity" held at Wolfgang Pauli Institute in Vienna, Austria in June 2006. D.P. is supported by the NSERC Discovery and PREA grants. P.G.K. is supported by NSF through the grants DMS-0204585, DMS-CAREER and DMS-0505663.

\section{References}

[1] I.V. Barashenkov, "Stability criterion for dark solitons", Phys. Rev. Lett. 77, 1193 (1996)

[2] I.V. Barashenkov and E.Y. Panova, "Stability nd evolution of the quiescient and travelling solitonic bubbles", Physica D 69, 114-134 (1993)

[3] M.D. Barrett, J.A. Sauer and M.S. Chapman, "All-optical formation of an atomic Bose-Einstein condensate", Phys. Rev. Lett. 87, 010404 (2001)

[4] A. de Bouard, "Instability of stationary bubbles", SIAM J. Math. Anal. 26, 566-582 (1995) 
[5] V.A. Brazhnyi and V.V. Konotop, "Evolution of a dark soliton in a parabolic potential: application to Bose-Einstein condensates", Phys. Rev. A 68, 043613 (2003)

[6] J.C. Bronski and R.L. Jerrard, "Soliton dynamics in a potential", Math. Res. Lett. 7, 329-342 (2000)

[7] L.D. Carr, M.J. Holland and B.A. Malomed, "Macroscopic quantum tunnelling of Bose-Einstein condensates in a finite potential well”, J. Phys. B: At. Mol. Opt. Phys. 38, 3217-3231 (2005)

[8] X.J. Chen, Z.D. Chen, and N.N. Huang, " A direct perturbation theory for dark solitons based on a complete set of the squared Jost solutions", J. Phys. A: Math. Gen. 31, 6929-6947 (1998)

[9] M. Chugunova and D. Pelinovsky, "Count of eigenvalues in the generalized eigenvalue problem", submitted to SIAM J. Math. Anal. (2006)

[10] E.A. Coddington and N. Levinson, " Theory of ordinary differential equations", (McGraw-Hill, New York, 1955)

[11] S. Cuccagna and D. Pelinovsky, "Bifurcations from the end points of the essential spectrum in the linearized NLS problem", J. Math. Phys. 46053520 (2005)

[12] B. Deconinck, D. Pelinovsky, and J.D. Carter, "Transverse instabilities of deep-water solitary waves", Proc. Roy. Soc. A 462, 2039-2061 (2006)

[13] J. Frhlich, S. Gustafson, B.L.G. Jonsson, and I.M. Sigal, "Solitary wave dynamics in an external potential", Comm. Math. Phys. 250, 613-642 (2004)

[14] P.D. Hislop and I.M. Sigal, Introduction to spectral theory with applications to Schrödinger operators, (Springer-Verlag, 1996)

[15] N.N. Huang, S. Chi, and X.J. Chen, "Foundations of direct perturbation method for dark solitons", J. Phys. A: Math. Gen. 32, 3939-3945 (1999)

[16] B.L.G. Jonsson, J. Fröhlich, S. Gustafson, and I.M. Sigal, "Long time motion of NLS solitary waves in a confining potential", Ann. Henri Poincare 7, 621-660 (2006)

[17] M. Johansson and Yu.S. Kivshar, "Discreteness-induced oscillatory instabilities of dark solitons", Phys. Rev. Lett. 82, 85 (1999)

[18] T. Kapitula, "Stability of waves in perturbed Hamiltonian systems", Physica D 156, 186-200 (2001)

[19] T. Kapitula and J. Rubin, "Existence and stability of standing hole solutions to complex GinzburgLandau equations", Nonlinearity 13, 77-112 (2000)

[20] T. Kapitula and B. Sandstede, "Eigenvalues and resonances using the Evans function", Discr. Cont. Dyn. Syst. B 10, 857-869 (2004)

[21] P.G. Kevrekidis and D.J. Frantzeskakis, "Pattern forming dynamical instabilities of Bose-Einstein Condensates", Mod. Phys. Lett. B 18, 173-202 (2004)

[22] Yu.S. Kivshar and B. Luther-Davies, "Dark optical solitons: physics and applications", Phys. Rep. 298, 81-197 (1998) 
[23] Yu.S. Kivshar and X. Yang, "Perturbation-induced dynamics of dark solitons", Phys. Rev. E 49, 1657-1670 (1994)

[24] V.V. Konotop and V.E. Vekslerchik, "Direct perturbation theory for dark solitons", Phys. Rev. E 49, 2397-2407 (1994)

[25] E.A. Kuznetsov and S.A. Turitsyn, "Instabilities and collapse of solitons in the medium with defocusing nonlinearity", Sov. Phys. JETP 67, 1583-1588 (1988)

[26] V.M. Lashkin, "Perturbation theory for dark solitons: Inverse scattering transform approach and radiative effects", Phys. Rev. E 70, 066620 (2004)

[27] J. Lega and S. Fauve, "Traveling hole solutions to the complex Ginzburg-Landau equation as perturbations of nonlinear Schrödinger dark solitons", Physica D 102, 234-252 (1997)

[28] Z. Lin, "Stability and instability of traveling solitonic bubbles", Adv. Diff. Eqs. 7, 897-918 (2002)

[29] M. Maris, "Stationary solutions to a nonlinear Schrödinger equation with potential in one dimension", Proc. Roy. Soc. Edinburgh A 133, 409-437 (2003)

[30] M. Maris, "Global branches of travelling waves to a Gross-Pitaevskii-Schrödinger system in one dimension", SIAM J. Math. Anal. (2006), to be published.

[31] L. Di Menza and C. Gallo, "The black solitons of one-dimensional NLS equations", Nonlinearity (2006), to be published.

[32] R. Onofrio, C. Raman, J.M. Vogels, J.R. Abo-Shaeer, A.P. Chikkatur and W. Ketterle, "Observation of superfluid flow in a Bose-Einstein gas", Phys. Rev. Lett. 85, 2228-2231 (2000)

[33] D.E. Pelinovsky and R.H.J. Grimshaw, "Asymptotic methods in soliton stability theory", in $A d$ vances in Fluid Mechanics Series, 12: Nonlinear Instability Analysis, edited by L.Debnath and S.R.Choudhury, (Computational Mechanics Publications, Southampton, Boston, 1997), pp. 245312

[34] D.E. Pelinovsky, D. Frantzeskakis, and P.G. Kevrekidis, "Oscillations of dark solitons in trapped Bose-Einstein condensates", Phys. Rev. E 72, 016615 (2005)

[35] D.E. Pelinovsky, Yu.S. Kivshar, and V.V. Afanasjev, "Instability-induced dynamics of dark solitons", Phys. Rev. E 54, 2015-2032 (1996)

[36] D.E. Pelinovsky, Yu.A. Stepanyants and Yu.S. Kivshar, "Self-focusing of plane dark solitons in nonlinear defocusing media", Phys.Rev.E 51, 5016-5026 (1995)

[37] D.E. Pelinovsky, A.A. Sukhorukov, and Yu. S. Kivshar, "Bifurcations and stability of gap solitons in periodic potentials", Phys. Rev. E 70, 036618 (2004)

[38] D. Pelinovsky and J. Yang, Stability analysis of embedded solitons in the generalized third-order NLS equation, Chaos 15, 037115 (2005)

[39] C.J. Pethick and H. Smith, Bose-Einstein condensation in dilute gases, Cambridge University Press (Cambridge, 2002) 
[40] L.P. Pitaevskii and S. Stringari, Bose-Einstein Condensation, Oxford University Press (Oxford, 2003)

[41] B. Prinari, M.J. Ablowitz, and G. Biondini, "Inverse scattering transform for the vector nonlinear Schrödinger equation with non-vanishing boundary conditions", preprint (2006)

[42] N.P. Proukakis, N.G. Parker, D.J. Frantzeskakis, and C.S. Adams, "Analogies between dark solitons in atomic Bose-Einstein condensates and optical systems", J. Opt. B: Quantum Semiclass. Opt. 6, S380-S391 (2004)

[43] C. Raman, M. Köhl, R. Onofrio, D.S. Durfee, C.E. Kuklewicz, Z. Hadzibabic and W. Ketterle, "Evidence for a critical velocity in a Bose-Einstein gas", Phys. Rev. Lett. 83, 2502-2505 (1999)

[44] B. Sandstede and A. Scheel, "Absolute instabilities of standing pulses", Nonlinearity 18, 331-378 (2005)

[45] B. Simon, "Resonances in one dimension and Fredholm determinants", J. Funct. Anal. 178, 396$420(2000)$

[46] P.E. Zhidkov, "Existence of solutions to the Cauchy problem and stability of kink-solutions of the nonlinear Schrödinger equation", Siberian. Math. J. 33, 239-246 (1992) 